\title{
Insights into Non-Covalent Interactions with a Machine-Learned Electron Density
}

Alberto Fabrizio, Andrea Grisafi, Benjamin Meyer, Michele Ceriotti, Clemence Corminboeuf

Submitted date: 03/06/2019 - Posted date: 04/06/2019

Licence: CC BY-NC-ND 4.0

Citation information: Fabrizio, Alberto; Grisafi, Andrea; Meyer, Benjamin; Ceriotti, Michele; Corminboeuf, Clemence (2019): Insights into Non-Covalent Interactions with a Machine-Learned Electron Density. ChemRxiv. Preprint.

Chemists continuously harvest the power of non-covalent interactions to control phenomena in both the microand macroscopic worlds. From the quantum chemical perspective, the strategies essentially rely upon an in-depth understanding of the physical origin of these interactions, the quantification of their magnitude and their visualization in real-space. The total electron density rho(r) represents the simplest yet most comprehensive piece of information available for fully characterizing bonding patterns and non-covalent interactions. The charge density of a molecule can be computed by solving the Schrodinger equation, but this approach becomes rapidly demanding if the electron density has to be evaluated for thousands of different molecules or for very large chemical systems, such as peptides and proteins. Here we present a transferable and scalable machine-learning model capable of predicting the total electron density directly from the atomic coordinates. The regression model is used to access qualitative and quantitative insights beyond the underlying $r h o(r)$ in a diverse ensemble of sidechain-sidechain dimers extracted from the BioFragment database (BFDb). The transferability of the model to more complex chemical systems is demonstrated by predicting and analyzing the electron density of a collection of 8 polypeptides.

File list (2)

ML_Rho_NCI.pdf (2.98 MiB)

view on ChemRxiv - download file

ML_Rho_NCl_SI.pdf (2.98 MiB)

view on ChemRxiv - download file 


\title{
Insights into non-covalent interactions with a machine-learned electron density
}

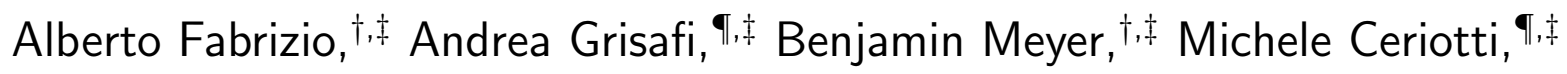 \\ and Clemence Corminboeuf*, $\uparrow, \ddagger$ \\ $\dagger$ †aboratory for Computational Molecular Design, Institute of Chemical Sciences and \\ Engineering,École Polytechnique Fédérale de Lausanne, CH-1015 Lausanne, Switzerland \\ $\ddagger$ National Centre for Computational Design and Discovery of Novel Materials (MARVEL), \\ École Polytechnique Fédérale de Lausanne, 1015 Lausanne, Switzerland \\ \Laboratory of Computational Science and Modeling, IMX, École Polytechnique Fédérale \\ de Lausanne, 1015 Lausanne, Switzerland \\ E-mail: clemence.corminboeuf@epfl.ch
}




\begin{abstract}
Chemists continuously harvest the power of non-covalent interactions to control phenomena in both the micro- and macroscopic worlds. From the quantum chemical perspective, the strategies essentially rely upon an in-depth understanding of the physical origin of these interactions, the quantification of their magnitude and their visualization in real-space. The total electron density $\rho(\boldsymbol{r})$ represents the simplest yet most comprehensive piece of information available for fully characterizing bonding patterns and non-covalent interactions. The charge density of a molecule can be computed by solving the Schrödinger equation, but this approach becomes rapidly demanding if the electron density has to be evaluated for thousands of different molecules or for very large chemical systems, such as peptides and proteins. Here we present a transferable and scalable machine-learning model capable of predicting the total electron density directly from the atomic coordinates. The regression model is used to access qualitative and quantitative insights beyond the underlying $\rho(\boldsymbol{r})$ in a diverse ensemble of sidechain-sidechain dimers extracted from the BioFragment database (BFDb). The transferability of the model to more complex chemical systems is demonstrated by predicting and analyzing the electron density of a collection of 8 polypeptides.
\end{abstract}

\title{
Graphical TOC Entry
}

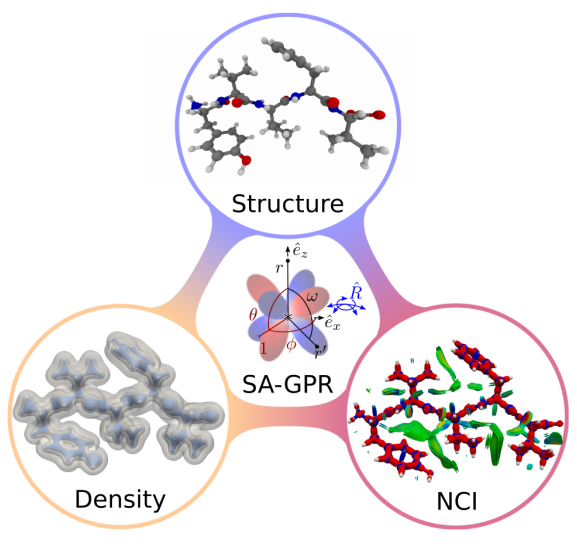




\section{Introduction}

Non-covalent interactions (NCIs) govern a multitude of chemical phenomena and are key components for constructing molecular architectures. ${ }^{1}$ Their importance fostered an intense research effort to accurately quantify their magnitude and develop an intuitive characterization of their physical nature using quantum chemistry. ${ }^{2-6}$ Among the different approaches to characterize non-covalent interactions, one of the simplest and most generally applicable takes as a starting point the electron density $\rho(\boldsymbol{r})$ that encodes, in principle, all the information needed to fully characterize a chemical system. ${ }^{7}$ Despite the fact that the universal functional relationship between total energy and $\rho(\boldsymbol{r})$ remains unknown, existing approximations within the framework of Kohn-Sham DFT $(\mathrm{KS}-\mathrm{DFT})^{8}$ do permit access to all molecular properties within a reasonable degree of accuracy. ${ }^{9-11}$

Properties that can be derived exactly from the electron density distribution include molecular and atomic electrostatic moments (e.g., charges, dipole, quadrupoles), electrostatic potentials and electrostatic interaction energies. Knowledge of these quantities is fundamental in diverse chemical applications, including the computation of the IR intensities, ${ }^{12}$ the identification of binding sites in host-guest compounds, ${ }^{13-15}$ and the exact treatment of electrostatics within molecular simulations. ${ }^{16}$ Moreover, analyzing the deformation of $\rho(\boldsymbol{r})$ in the presence of an external field provides access to another set of fundamental properties, namely molecular static (hyper)polarizabilities and, thus, to the computation of Raman spectra $^{17}$ and non-linear optical properties. ${ }^{18-21}$

The natural representation of the electron density in real space makes it especially suitable for accessing spatial information about structural and electronic molecular properties, including X-Ray structure refinement ${ }^{22-27}$ and representations using scalar fields. ${ }^{6}$ Routinely

used examples include the quantum theory of atoms in molecules (QTAIM), ${ }^{28,29}$ the density overlap region indicator (DORI), ${ }^{30}$ and the non-covalent interaction (NCI) index. ${ }^{31,32}$

$\rho(\boldsymbol{r})$ is generally obtained by solving the electronic structure problem through ab-initio computations. The main advantage of this approach is that it returns the variationally op- 
timized electronic density for a given Hamiltonian. Yet, ab-initio computations can become increasingly burdensome if $\rho(\boldsymbol{r})$ has to be evaluated for thousands of different molecules or for very large chemical systems, such as peptides and proteins. These large scale problems are typically tackled using a more scalable approach that consists of either using linear scaling techniques such as Mezey's molecular electron density LEGO assembler (MEDLA) ${ }^{33,34}$ and adjustable density matrix assembler (ADMA), ${ }^{35-37}$ as well as approaches based on localized molecular orbitals, such as ELMO. ${ }^{38-41}$ Another methodology belonging to this second category involves the use of experimental techniques, such as X-Ray diffraction, to probe the electron density and subsequently reconstructing $\rho(\boldsymbol{r})$ through multipolar models ${ }^{42-44}$ and pseudo-atomic libraries, such as ELMAM, ${ }^{45-48}$ ELMAM2,${ }^{49,50}$ UBDB, ${ }^{51,52}$ Invarioms $^{53}$ and SBFA. ${ }^{54}$ While successful, these two methodologies have intrinsic limits: the first is unable to capture the deformations of the charge density due to intermolecular interactions unless a suitable fragment is generated $a d-h o c$, while the second relies on experimental data and is difficult to extend to thousands of different chemical systems at once. Recently, the development of several machine-learning models targeting the electron density has effectively established a third promising methodology, with the potential to overcome the limitations of the more traditional approaches.

The first machine-learning model of $\rho(\boldsymbol{r})$ was developed on the basis of the HohenbergKohn mapping between the nuclear potential and the electron density. ${ }^{55,56}$ Although successful, the choice of the nuclear potential as a representation of the different molecular conformations and the expansion of the electron density in an orthogonal plane-wave basis effectively constrained this landmark model to relatively small and rigid molecules with limited transferability to larger systems. Recently, we proposed an atom-centered, symmetryadapted Gaussian process regression ${ }^{57}$ (SA-GPR) framework explicitly targeting the learning of the electron density. ${ }^{58}$ Using an optimized non-orthogonal basis set, pseudo-valence electron densities could be predicted in a linear-scaling and transferable manner, meaning that the model is able to tackle much larger chemical systems than the those used to train the 
regression model. A third approach, that can also achieve transferability between different systems, uses a direct grid-based representation of the atomic environment to learn and predict the electronic density in each point of the molecular space. ${ }^{59-61}$ Representing the density field on a large set of grids points rather than on a basis set effectively avoids the introduction of a basis set error, but also dramatically increases the computational effort.

One should also consider that machine learning, being a data-driven approach, requires high-quality, diverse reference data. Fortunately, several specialized benchmark databases that target NCIs have appeared over the past decade. From the original S22 ${ }^{62}$ to NCIE53, ${ }^{63}$ S66, ${ }^{64}$ NBC10ext ${ }^{65,66}$ and S12L,${ }^{67}$ the evolution of these datasets has, generally, followed a prescription of increasing the number of entries, principally by including subtler interactions and/or larger systems. In this respect, the databases of Friesner, ${ }^{68}$ Head-Gordon ${ }^{69}$ and the recent $\mathrm{BFDb}$ of Sherrill, ${ }^{70}$ constitute a special category because of their exceptional size (reaching thousands of entries) which are now sufficiently large to be compatible with machine-learning applications. Beyond their conceptual differences, each of these benchmark sets aim at improving the capability of electronic structure methods to describe the energetic aspects of non-covalent interactions.

In this work, we introduce a dramatic improvement of our previous density-learning approach by making the regression machinery of $\rho(\boldsymbol{r})$ compatible with density-fitting auxiliary basis sets. These specialized basis sets are routinely used in quantum chemistry to approximate two-center one-electron densities. Here, the auxiliary basis sets are used directly to represent the electron densities that enter our machine-learning model, with the additional advantage of avoiding the arbitrary basis set optimization procedures on the machine-learning side. This enhanced framework leverages the transferability of our symmetry-adapted regression method and is capable of learning the all-electron density across a vast spectrum of 2291 chemically diverse dimers formed by sidechain-sidechain interactions extracted from the BioFragment Database (BFDb). ${ }^{70}$ The performance of the method is demonstrated through the reproduction of $\rho(\boldsymbol{r})$ between and within each monomer forming the dimers. The ac- 
curacy of the predicted densities is assessed by computing density-based scalar fields and electrostatic potentials, while the errors made with respect to the reference densities are computed by direct integration on three-dimensional grids. As a major breakthrough, the model is used to predict the charge density of a set of 8 polypeptides $(\sim 100$ atoms $)$ at DFT accuracy in few minutes.

\section{Methods}

Gaussian process regression (GPR) can be extended to encode all the fundamental symmetries of the $O(3)$ group, effectively allowing machine-learning of all the molecular properties that transform as spherical tensors under rotation and inversion operations. ${ }^{57,71}$ In the specific case of the electron density, the scheme relies upon the decomposition of the field into additive, atom-centered contributions and the subsequent prediction of the corresponding expansion coefficients. ${ }^{58}$ In SA-GPR, each molecule is represented as a collection of atom-centered environments, whose relationships and similarities are measured by symmetry adapted kernels. An in-depth discussion about how a symmetry adapted regression model of the electron density can be constructed is reported in the supplementary materials.

The decomposition of the electron density in continuous atom-centered basis functions is the cornerstone of the scalability and transferability of our SA-GPR model. Beside being generally desirable, these properties are actually crucial to accurately describe the chemical diversity present in the BioFragment Database within a reasonable computational cost. On the other hand, the projection of the density field onto a basis set leads to an additional error on top of that which can be ascribed to machine learning. In practice, all the efforts placed into achieving a negligible machine-learning error are futile if the overall accuracy of the model is dictated by a large basis set decomposition error.

Standard quantum chemical basis sets are generally optimized to closely reproduce the behavior of atomic orbitals ${ }^{72}$ and results in unacceptable errors if used to decompose the 
electronic density (Figure 1). In contrast, specialized basis sets used in the density fitting approximation (also known as resolution-of-the-identity (RI) approximation) ${ }^{73-79}$ are specifically optimized to represent a linear expansion of one-electron charge densities obtained from the product of atomic orbitals. Using the RI-auxiliary basis sets $\left\{\phi_{k}^{R I}\right\}$, the total electron density field can be expressed as:

$$
\rho(\boldsymbol{r})=\sum_{k}^{N_{a u x}}\left(\sum_{a b}^{N_{A O}} D_{a b} d_{k}^{a b}\right) \phi_{k}^{R I}(\boldsymbol{r})=\sum_{k}^{N_{a u x}} c_{k} \phi_{k}^{R I}(\boldsymbol{r})
$$

where, $D_{a b}$ is the one-electron reduced density matrix and $d_{k}^{a b}$ are the RI-expansion coefficients.

Given a molecular geometry, the value of the basis functions can be readily computed at each point of space, leaving the $c_{k}$ expansion coefficients as the only ingredient needed to fully determine the amplitude of $\rho(\boldsymbol{r})$. In this sense, the prediction of the electron density by the SA-GPR model can be summarized as the prediction of the $c_{k}$ coefficients after training on a set of previously computed reference densities (details in the ESI).

As shown in Figure 1, the use of the RI-auxiliary basis sets results in nearly two orders of magnitude increase in the overall accuracy with respect to the corresponding standard basis set. The addition of diffuse functions marginally improves the performance of the decomposition, but leads to instabilities of the overlap matrix (high condition number) and increases dramatically the number of basis functions per atom. 

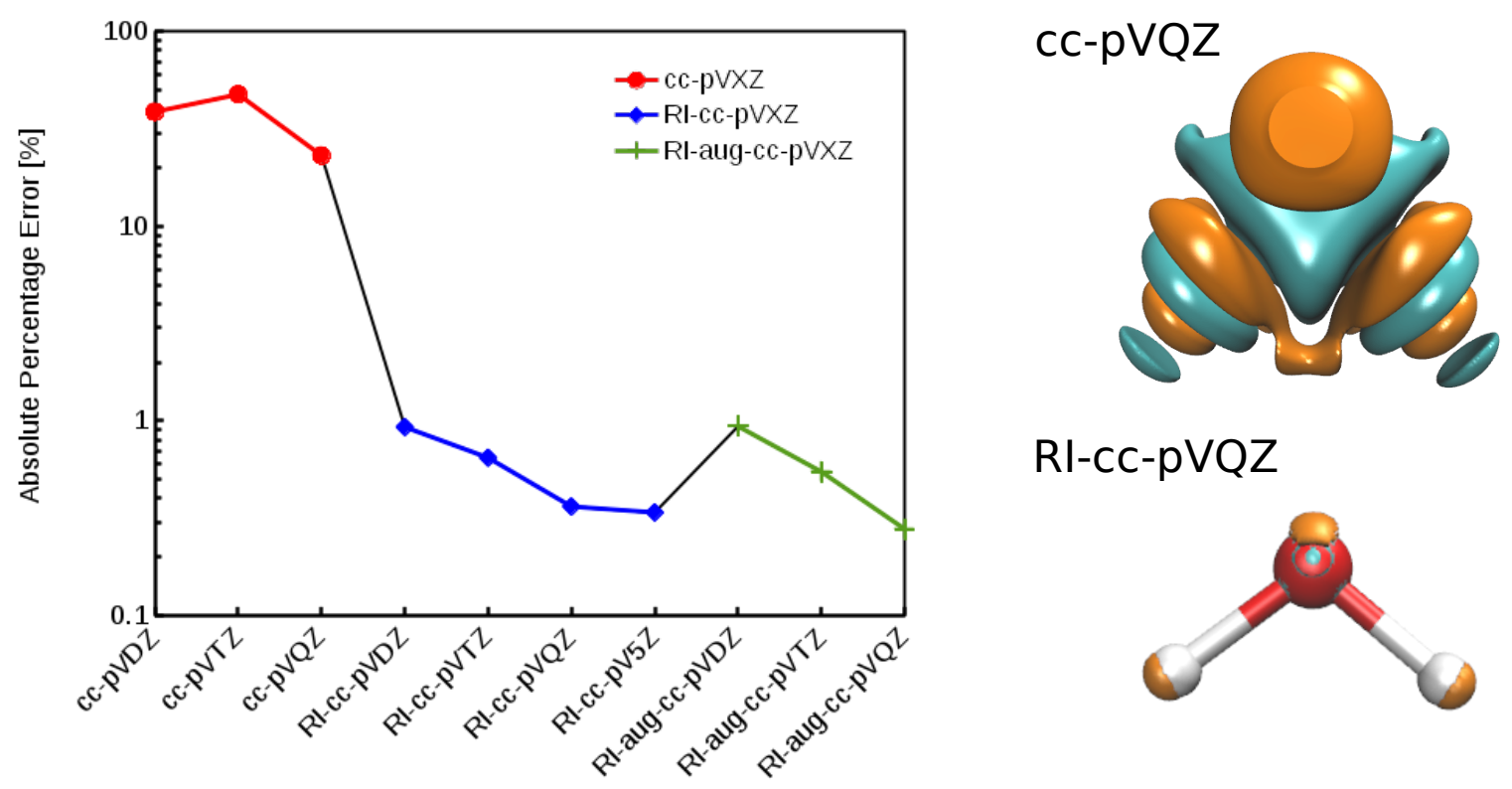

RI-cc-pVQZ

Figure 1: (left) Electron density of a single water molecule: evolution of the absolute percentage error depending on the choice of decomposition basis set. (right) Comparison of the density error made with the standard and the RI-auxiliary cc-pVQZ basis set ( cyan and orange isosurfaces refer to an error of $\pm 0.005 \mathrm{Bohr}^{-3}$ ). Reference density: PBE/cc-pVQZ.

In practice, Weigend's JK-fit cc-pVQZ ${ }^{78}$ basis set (henceforth: RI-cc-pVQZ) offers the best trade-off between accuracy and computational demand and therefore represents the best choice for the density decomposition.

\section{Computational Details}

The dataset of molecular dimers has been selected from the side-chain side-chain interaction (SSI) subset of the BioFragment Database (BFDb). ${ }^{70}$ The original set is made of 3558 dimers formed by amino-acids side-chain fragments taken from 47 different protein structures. Dimers with more than 25 atoms as well as those containing sulfur atoms were not considered. While the total number of sulfur-containing structures is too small to enable the machinelearning model to accurately capture its rich chemistry, the inclusion of the larger systems does not increase dramatically the chemical diversity of the dataset. The final dataset contains a total of 2291 dimers. 
As shown in Figure 2, the complete set of 2291 dimers spans a large variety of dominant interaction types, ranging from purely dispersion dominated complexes (in blue) to mixedinfluence (green and yellow) to hydrogen-bonded and charged systems (red). We retain the same classification criteria as in the original database to attribute the nature of the dominant interaction.

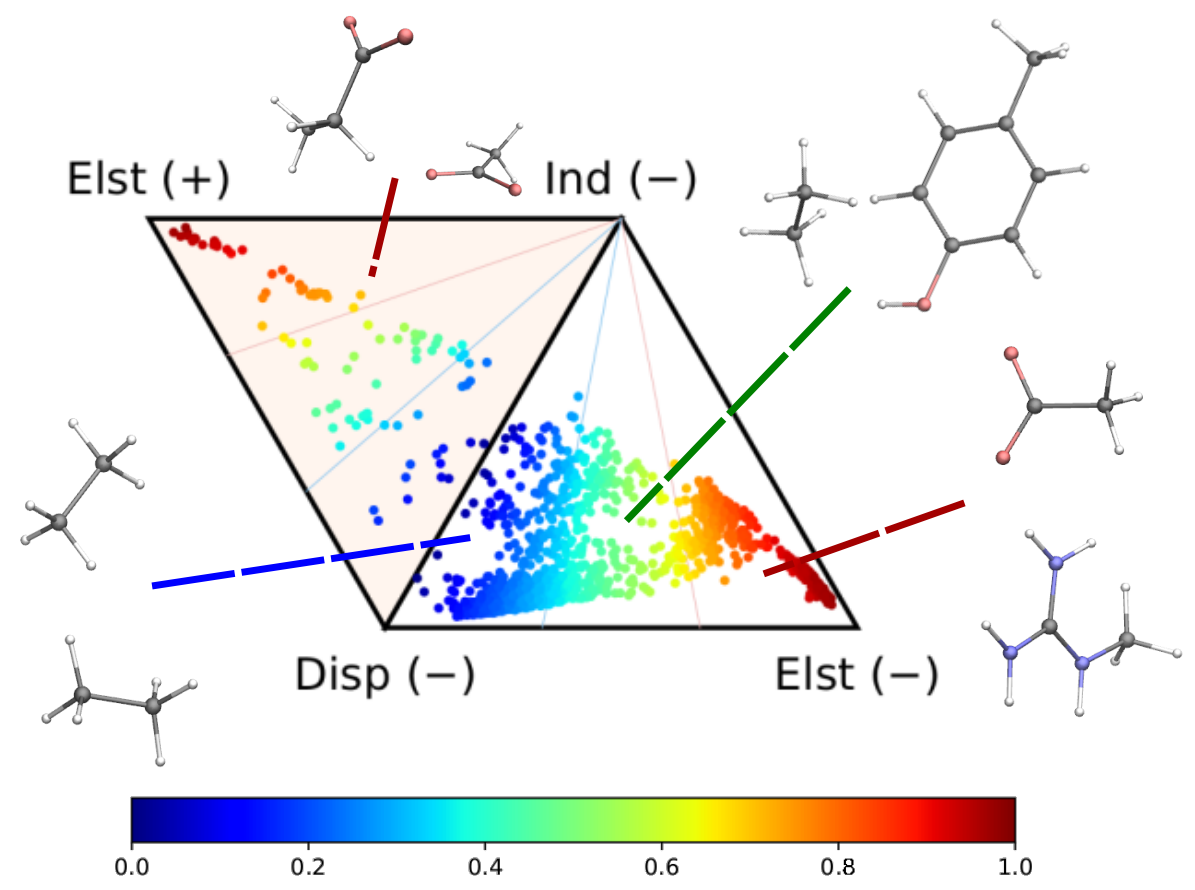

Figure 2: Ternary diagram representation of the attractive components of the dimer interaction energies for the 2291 systems considered in this work. The values of the SAPT analysis are taken from Ref. 70.

For each dimer, the reference full-electron density has been computed at the $\omega$ B97X-D/ccpVQZ level using the resolution of identity approximation for the Coulomb and exchange potential (RI-JK). This implies that RI-auxiliary functions up to $l=5$ are included for carbon, nitrogen and oxygen atoms while auxiliary functions up to $l=4$ are used for hydrogen atoms. 


\section{Results and Discussion}

The training set for the density-learning model was chosen by randomly picking 2000 dimers out of a total of 2291 possibilities. The remaining 291 were used to test the accuracy of the predictions. Given the tremendous number of possible atomic environments $(\sim 40000)$ associated with such a chemically diverse database, a subset of $M$ reference environments was selected to reduce the dimensionality of the regression problem (see Supplementary information). To assess the consequences of this dimensionality reduction, the learning exercise was performed on three different sizes $M=\{100,500,1000\}$ for the reference atomic environments. Figure 3 summarizes the performance of the machine learning algorithm, expressed in terms of the mean absolute difference between the predicted and ab-initio densities reconstructed on the RI auxiliary-basis, and weighted by the total number of electrons of each dimer.
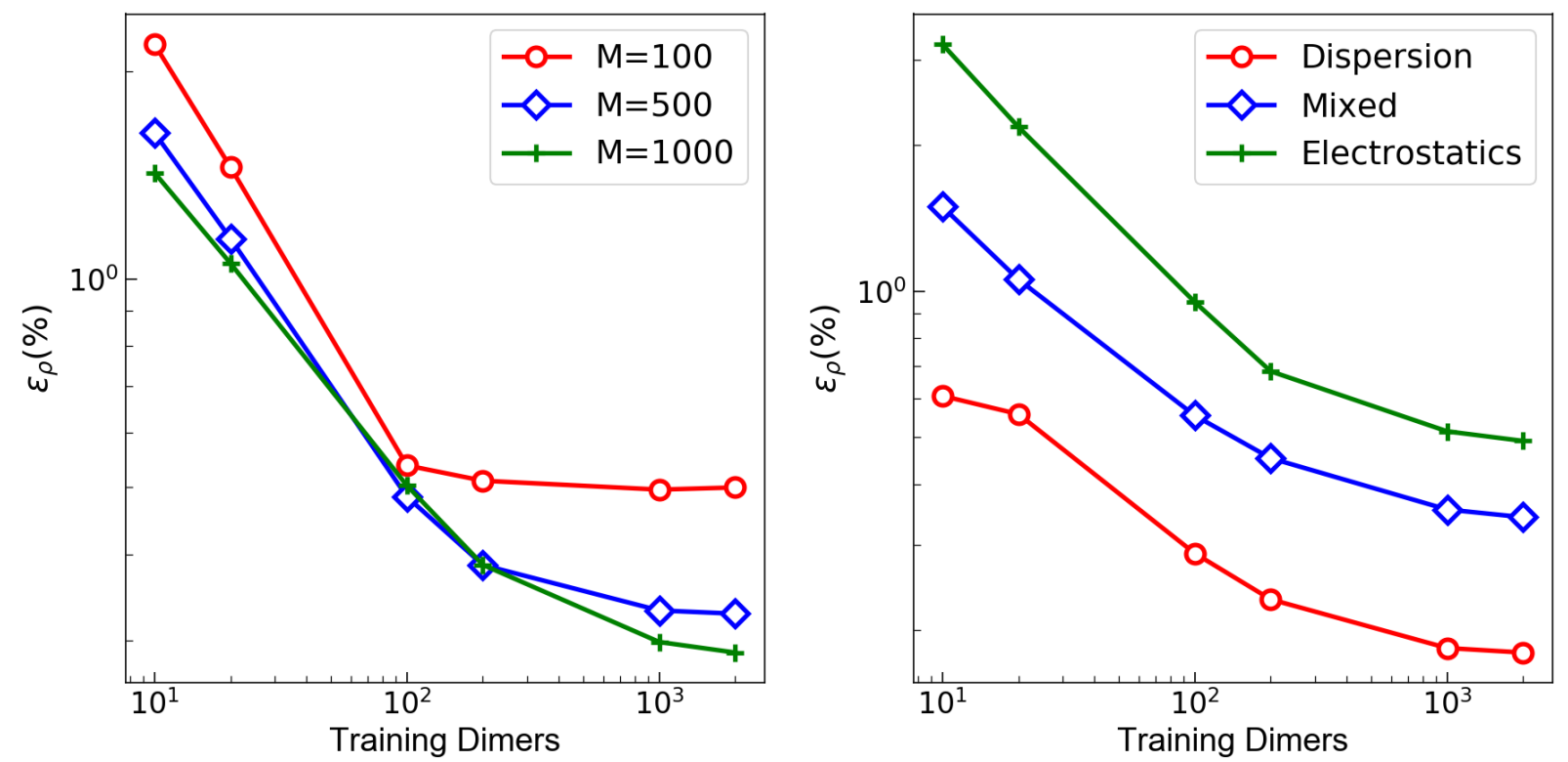

Figure 3: Learning curves. (left) weighted mean absolute percentage error $\left(\epsilon_{\rho}(\%)\right)$ of the predicted SA-GPR densities as a function of the number of training dimers. The weights correspond to the number of electrons in each dimer and the normalization is defined by the total number of electrons. Color code reflects the number of reference environments. (right) $\epsilon_{\rho}(\%)$ of the predicted SA-GPR densities $(\mathrm{M}=1000)$ divided per dominant contribution to the interaction energy according to Ref. [ 70]. $\epsilon_{\rho}(\%)=100 \times \frac{1}{N_{\mathrm{e}}} \sum_{i} N_{\mathrm{e}}^{i} \frac{\int d \boldsymbol{r}\left|\rho_{\mathrm{QM}}^{i}(\boldsymbol{r})-\rho_{\mathrm{ML}}^{i}(\boldsymbol{r})\right|}{\int d \boldsymbol{r} \rho_{\mathrm{QM}}^{i}(\boldsymbol{r})}$. 
As shown in the first panel of Figure 3, 100 training dimers were sufficient to reach saturation of the density error around $0.5 \%$ for $M=100$. This result already outperforms the level of accuracy reached in our previous work, which is remarkable given the large chemical diversity of the dataset and the consideration of all-electron densities. Learning curves obtained with $M=500$ and $M=1000$ show steeper slopes, approaching saturation at about 2000 training dimers with errors that were reduced to $\sim 0.2-0.3 \%$. The predicted full-electron densities are five times more accurate than the previous predictions of valence-only densities (approximately 1\%). ${ }^{58} \mathrm{~A}$ more detailed analysis of the $M=1000$ learning curve reveals a strong dependence on the nature of the dominant interaction (Figure 3). Specifically, stronger non-local character in the interaction yields a larger error. This is especially prevalent for dimers dominated by electrostatic interactions (i.e., hydrogen bonds, charged systems), which are characterized by errors that are twice as large as those found in other regimes.

The origin of this slow convergence arises from two factors. First, only about $20 \%$ of the dimers are dominantly bound by electrostatics. ${ }^{70}$ The priority of the regression model is thus to minimize the error on the other classes. Second, there is a fundamental dichotomy between the local nature of our symmetry-adapted learning scheme and the long-range nature of the interactions. In this respect, a global ML representation of the density field would be more suitable, but this would imply renouncing the scalability and transferability of the model. Given a large enough training set, however, our SA-GPR model is able to capture the density deformations due to the field generated by the neighboring molecule. The reason is rooted in the intrinsic locality of density deformations and in the concept of "nearsightedness" 80,81 of all local electronic properties, which constitutes a theoretical justification for a local decomposition of such quantities.

The fundamental advantage of setting the electron density as the machine-learning target is the broad spectrum of chemical properties that are directly derivable from $\rho(\boldsymbol{r})$. For instance, the predicted charge densities are the key ingredient in density-dependent scalar fields aimed at visualizing and characterizing interactions between atoms and molecules in 
real space. Examples of the density overlap region indicator (DORI) ${ }^{30}$ are given in Figure 4 for representative dimers. Compared to the rather featureless $\rho(\boldsymbol{r})$, DORI reveals fine details of electronic structure, which constitute a more sensitive probe for the quality of the machine-learning predictions. In particular, it reveals density overlaps (or clashes) associated with bonding and non-covalent regions on equal footing through the behavior of the local wave-vector $(\nabla \rho(\boldsymbol{r}) / \rho(\boldsymbol{r})) .{ }^{82-84}$

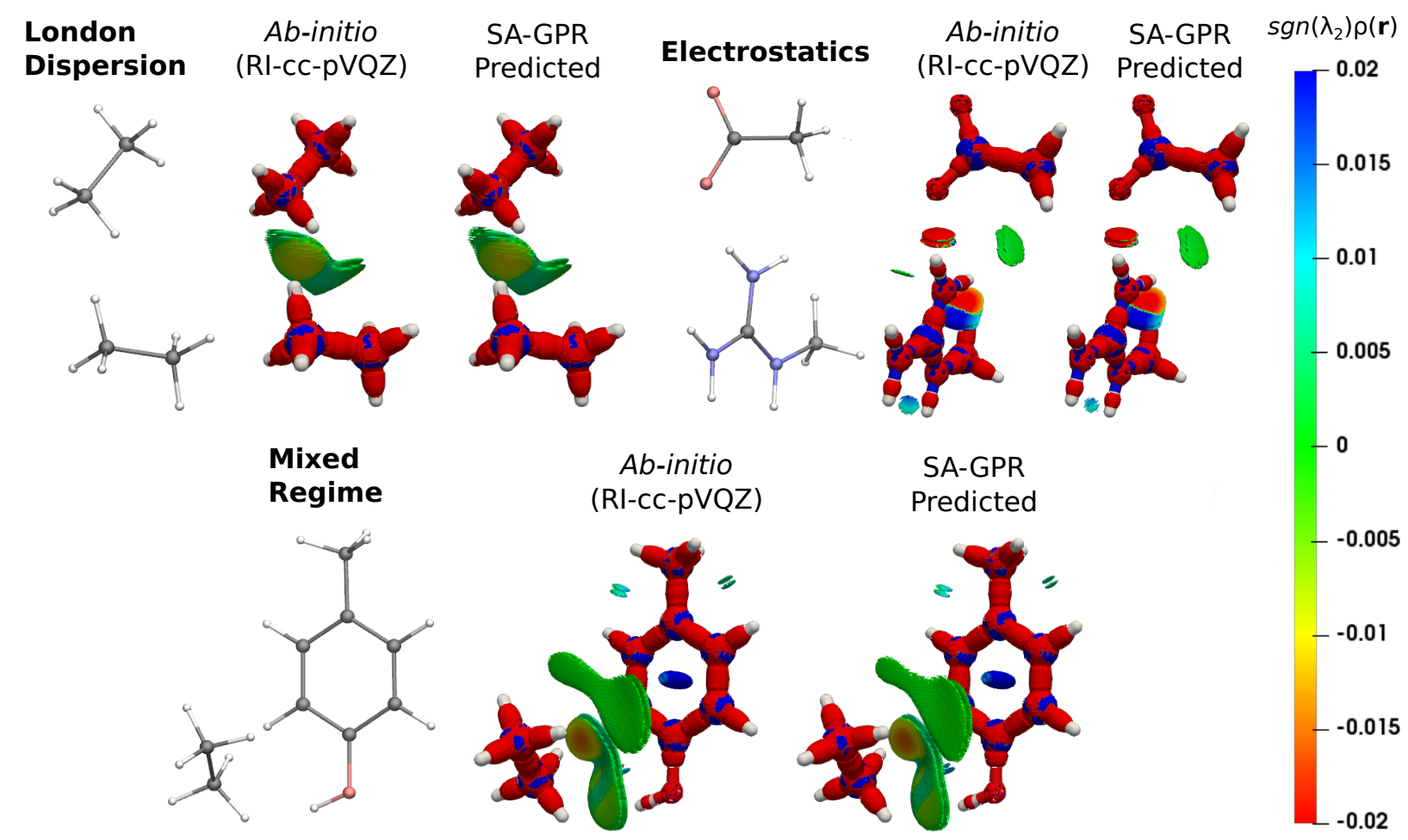

Figure 4: DORI maps of representative dimers for each type of dominant interaction (DORI isovalue: 0.9). Isosurfaces are color-coded ${ }^{31}$ with $\operatorname{sgn}\left(\lambda_{2}\right) \rho(\boldsymbol{r})$ in the range from attractive -0.02 a.u. (red) to repulsive 0.02 a.u. (blue). In particular, $\operatorname{sgn}\left(\lambda_{2}\right) \rho(\boldsymbol{r})<0$ characterizes covalent bonds or strongly attractive NCIs (e.g. H-bonds); $\operatorname{sgn}\left(\lambda_{2}\right) \rho(\boldsymbol{r}) \sim 0$ indicates weak attractive interactions (van der Waals); $\operatorname{sgn}\left(\lambda_{2}\right) \rho(\boldsymbol{r})>0$ repulsive NCIs (e.g. steric clashes).

As shown in Figure 4, the intra- and intermolecular DORI domains obtained with the SA-GPR densities are indistinguishable from those in the ab-initio maps. This performance is especially impressive for the density clashes associated with low density values, as is typical for the non-covalent domains. All the features are well captured by the predicted densities 
ranging from large and delocalized basins typical of the van der Waals complexes (in green) to the compact and directional domains typical of electrostatic interactions, to intramolecular steric clashes (e.g. phenol, mixed regime). Overall, these results illustrate that the residual $0.2 \%$ mean absolute percentage error does not significantly affect the density amplitude in the valence and intermolecular regions that are accurately described by the SA-GPR model. The highest amplitude errors are concentrated near the nuclei in the region dominated by the core-density fluctuations.

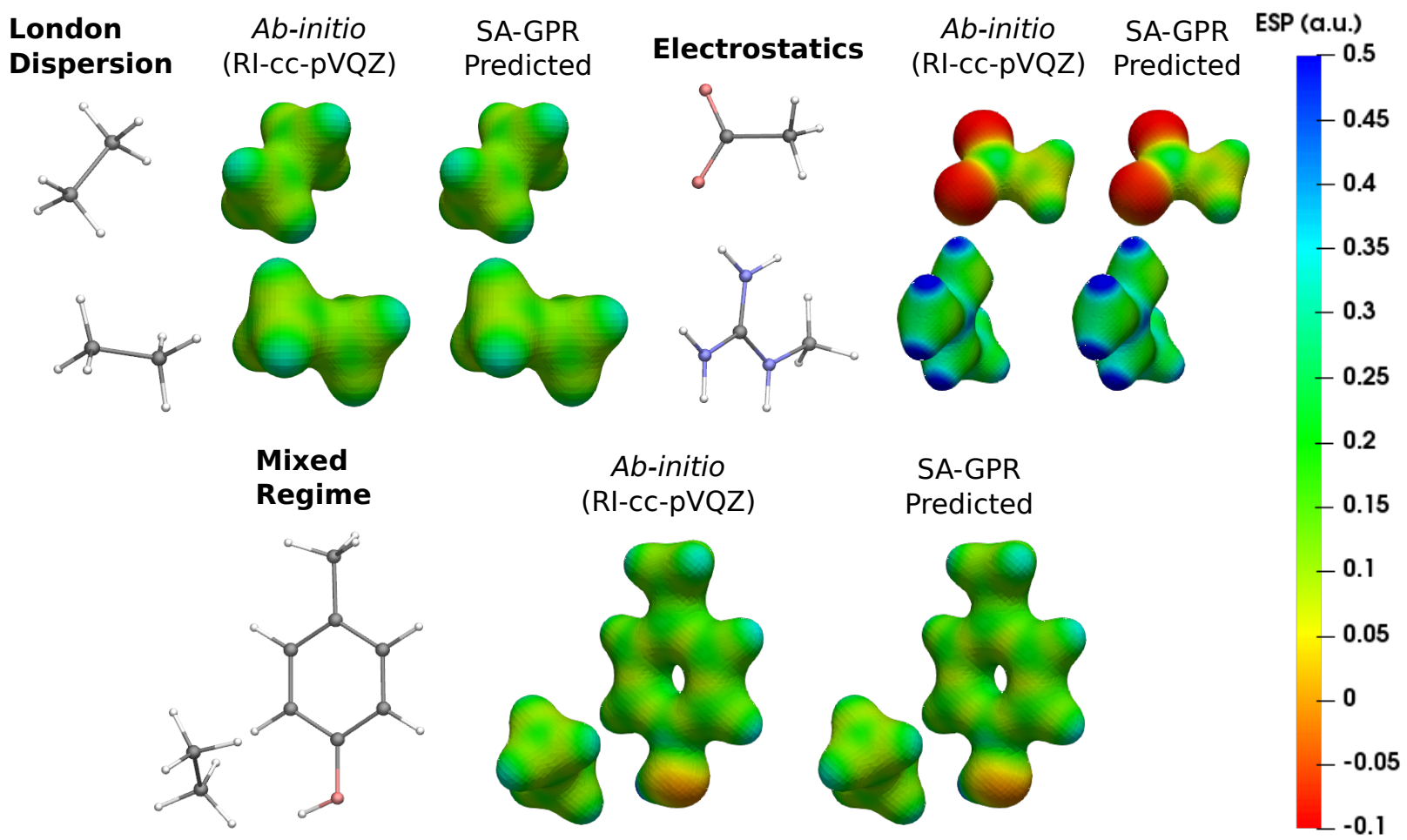

Figure 5: Electrostatic potential (ESP) maps of representative dimers for each type of dominant interaction (density isovalue: $0.05 \mathrm{e}^{-} \mathrm{Bohr}^{-3}$ ). ESP potential is given in Hartree atomic units (a.u.).

The versatility of the machine-learning prediction is further illustrated by using the predicted densities to compute the molecular electrostatic potential (ESP) for the same representative dimers (Figure 5). ESP maps based on predicted densities agree quantitatively with the ab-initio reference and correctly attribute the sign and magnitude of the electrostatic potential in all regions of space. Importantly, the accuracy of the ESP magnitude 
remains largely independent of the dominant interaction type. This is especially relevant for charged dimers (electrostatics) as it demonstrates that despite slower convergence of the learning curve for this category, the achieved accuracy of the model is sufficient to describe the key features of the electrostatic potential.

The most widespread applications of ESP maps exploit qualitative information (e.g., identification of the molecular regions most prone to electrophilic/nucleophilic attack) but the electrostatic potentials can be related to quantitative properties such as the degree of acidity of hydrogen bonds and the magnitude of binding energies. ${ }^{85-89}$ As a concrete example related to structure-based drug design, we used a recent model that estimates the strength of the stacking interactions between heterocycles and aromatic amino acid side-chains directly from the ESP maps. ${ }^{87,88,90}$ This model derives the stacking energies of drug-like heterocycles from the maximum and mean value of their ESP within a surface delimited by molecular van der Waals volume (at $3.25 \AA$ above the molecular plane). ${ }^{87}$ Following this procedure, we used the ESP derived from the ML predicted densities to compute the binding energies between a representative heterocycle included in our dataset, the tryptophan side-chain, and the three aromatic amino acid side-chains (Figure 6). 


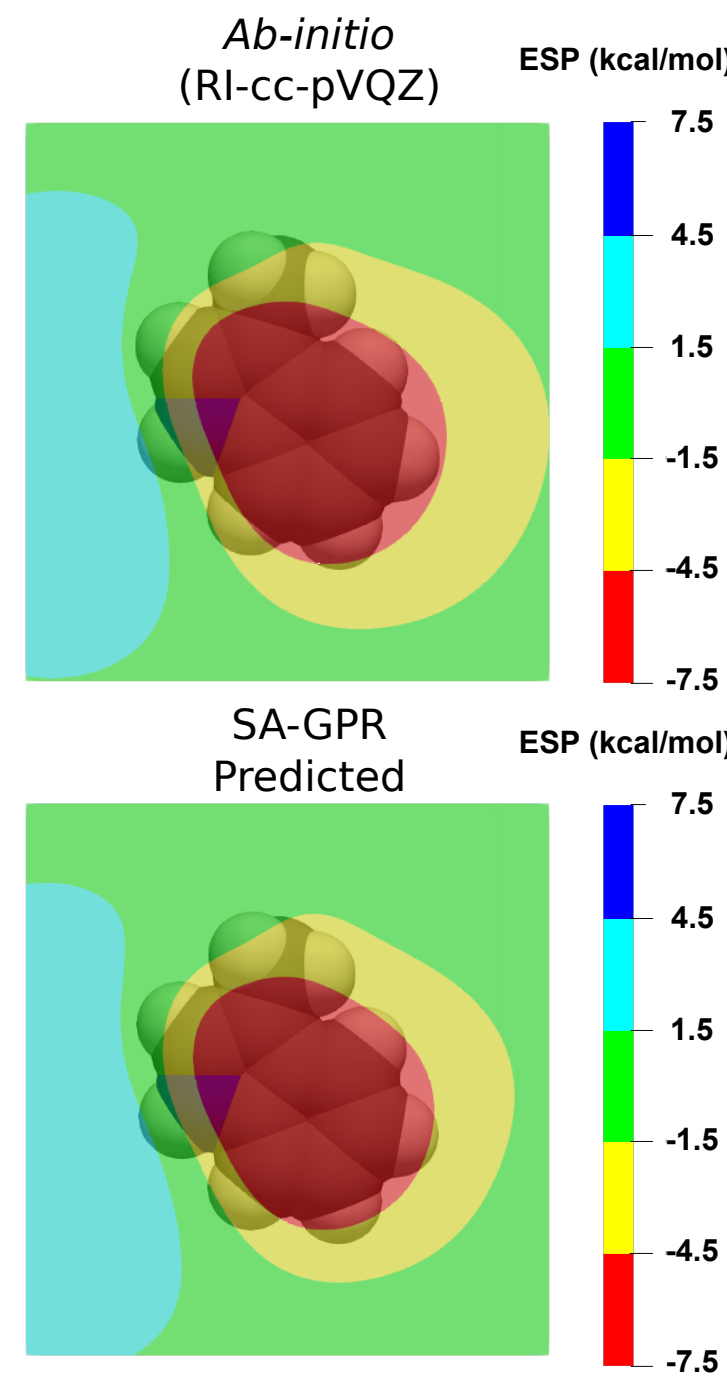

Ab-Initio Stacking Interaction Energy

PHE

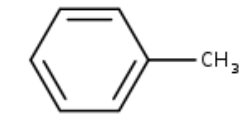

$-8.35 \mathrm{kcal} / \mathrm{mol}$

TYR

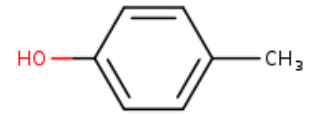

$-9.30 \mathrm{kcal} / \mathrm{mol}$

TRP<smiles>Cc1c[nH]c2ccccc12</smiles>

$-11.21 \mathrm{kcal} / \mathrm{mol}$

Predicted Stacking Interaction Energy

PHE

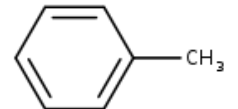

$-8.39 \mathrm{kcal} / \mathrm{mol}$

TYR

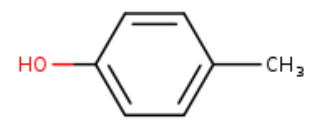

$-9.35 \mathrm{kcal} / \mathrm{mol}$

TRP<smiles>Cc1c[nH]c2ccccc12</smiles>

$-11.28 \mathrm{kcal} / \mathrm{mol}$

Figure 6: (left) Electrostatic potential maps $3.25 \AA$ above the plane of the tryptophan (TRP) side-chain. The van der Waals volume of TRP is represented in transparency. (Right) Stacking interaction energies of TRP with the phenylalanine (PHE), tyrosin (TYR) and tryptophan (TRP) side-chains computed as detailed in Ref. 87.

Comparison between $a b$-initio and ML predicted stacking interaction energies shows that the deviations in the ESP maps lead to minor errors on the order of $0.05 \mathrm{kcal} / \mathrm{mol}$. The largest deviations in the ESP would appear further away from the molecule, beyond the region exploited for the computation of the energy descriptors (i.e., the sum of the atomic van der Waals radii). This behavior is related to the propagation of the deviations in the density predictions $\delta \rho(\boldsymbol{r})$ to the electrostatic potential $\delta \hat{\phi}(\boldsymbol{k})=4 \pi \delta \hat{\rho}(\boldsymbol{k}) / k^{2}$. In particular, the error on the low frequency components of $\rho(\boldsymbol{r})$ is greatly enhanced as $k \rightarrow 0$, resulting 
in larger errors as the distance from the nuclei increases.

\section{Prediction on Polypeptides}

The tremendous advantage of the atom-centered density decomposition is to deliver a machinelearning model that depends only on the different atomic environments and not on the identity of the molecules included in the training set. Thanks to its transferability, the model provides access to density information of large macromolecules, at the sole price of including sufficient diversity, that can capture the chemical complexity of a larger system. The predictive power of this extrapolation procedure is demonstrated by using the machine-learning model exclusively trained on the $2291 \mathrm{BFDb}$ dimers to predict the electron density of 8 polypeptides taken from the Protein DataBank (PDB). ${ }^{91}$ The performance of the ML model for each macromolecules, labelled by their PBD ID, is reported in Figure 7.

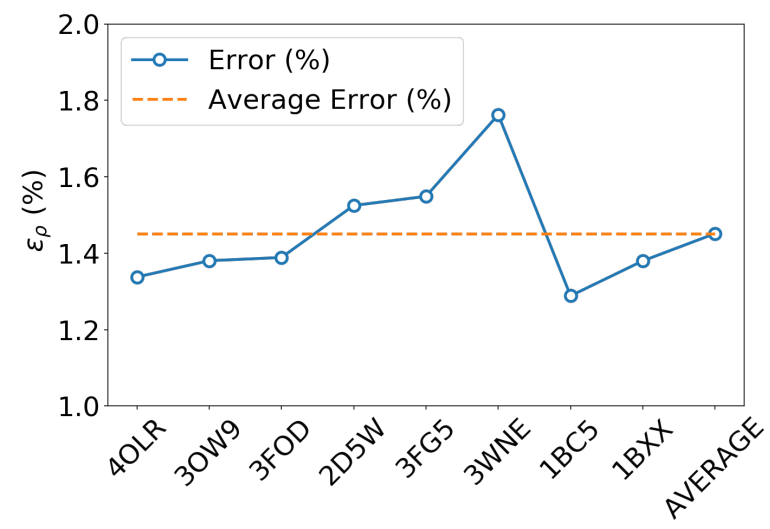

Figure 7: Weighted mean absolute percentage error $\left(\epsilon_{\rho}(\%)\right)$ of the predicted densities extrapolated for 8 biologically relevant peptides (protein databank ID).

Overall, the predictions lead to a low average error of only $1.5 \%$ for the 8 polypeptides, which is in line with the highest density errors obtained on the BFDb test set. Relevantly, the largest discrepancies are obtained for 3WNE, which is the only cyclopeptide of the set. The origin of these differences can be understood by performing a more detailed analysis on a representative polypeptide, the leu-enkephalin (4OLR). The errors in this percentage range 
do not affect the density-based properties, such as the spatial analysis of the intramolecular interactions with scalar fields (Figure 8 top right panel). Yet, most of the discrepancies occur along the amino acid backbone (Figure 8 lower panels), which is especially sensitive for the more strained 3WNE cyclopeptide.
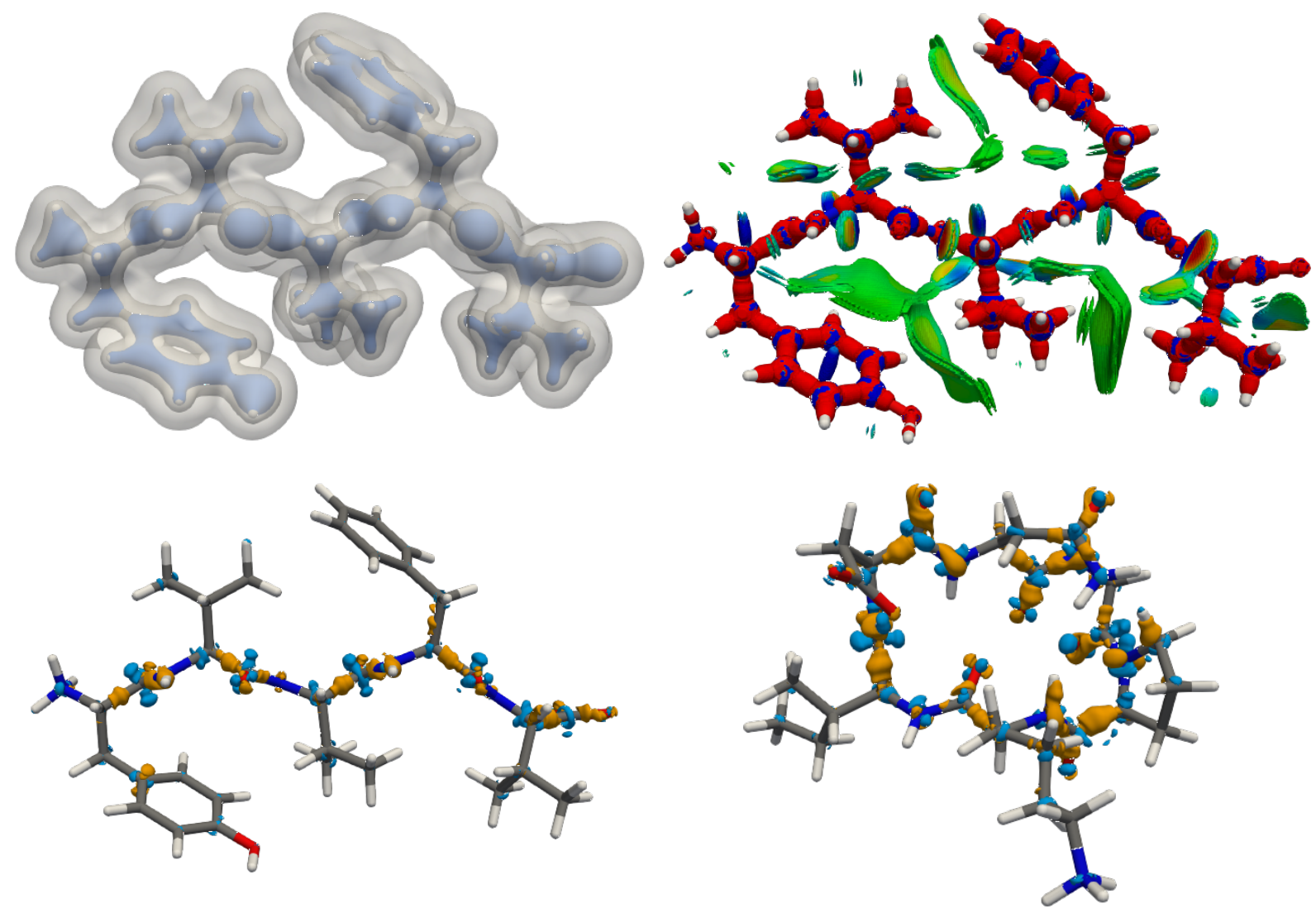

Figure 8: (top left) predicted electron density of enkephalin (PBD ID: 4OLR) at three isovalues: $0.25,0.1$, and $0.01 \mathrm{e}^{-} \mathrm{Bohr}^{-3}$. (top right) DORI map of enkephalin (DORI isovalue: 0.9 ) colored by $\operatorname{sgn}\left(\lambda_{2}\right) \rho(\boldsymbol{r})$ in the range from -0.02 a.u. (red) to 0.02 a.u. (blue) (lower left) density difference between predicted and ab-initio electron density (isovalues $\pm 0.01 \mathrm{e}^{-}$Bohr $\left.^{-3}\right)$. (lower right) density difference between predicted and ab-initio electron density of $3 \mathrm{WNE}$ (isovalues $\pm 0.01 \mathrm{e}^{-} \mathrm{Bohr}^{-3}$ ).

Although similar chemical environments were included in the training set, the error is determined by the lack of an explicit peptide bond motif and cyclopeptides in the training set. While this limitation could be addressed by ad hoc modification of the training set, the overall performance of the machine-learning model is rather exceptional as it provides in only a few minutes electron densities of DFT quality for large and complex molecular 
systems.

\section{Conclusion}

Given its central role in electronic structure methods, the total electron density is a very promising target for machine learning, since accurate predictions of $\rho(\mathbf{r})$ give access to all the information needed to characterize a chemical system. Among the many possible properties that can be computed from the electron density, the patterns arising from non-covalent interactions constitute a particular challenge for machine learning models owing to their longrange nature and subtle physical origin. An effective ML model should be transferable across different systems, efficient in learning from relatively small training sets, and accurate in predicting a $\rho(\mathbf{r})$ both in the quickly-varying region around the atomic nuclei, in the tail and - crucially for the study of non-covalent interactions - that are highly non-local and manifest in real space as slowing-varying regions with low densities. In this work we have presented a model that fulfills all of these requirements, based on an atom-centered decomposition of the density that uses an accurate resolution-of-identity basis set, a symmetry-adapted Gaussian Process regression ML scheme, and training on a diverse database of 2000 sidechain sidechain dimers extracted from the biofragment database.

The model reaches a $0.3 \%$ accuracy on a validation set, that is sufficient to investigate density-based fingerprints of NCIs, and to evaluate the electrostatic potential with sufficient accuracy to quantitatively estimate residue-residue interactions. The transferability of the model is demonstrated by predicting, at a cost that is orders of magnitude smaller than by explicit electronic structure calculations, the electron density for a demonstrative set of oligopeptides, with an accuracy sufficient to reliably visualize bonding patterns and noncovalent domains using the DORI scalar field. Even though the model reaches an impressive

accuracy (0.5\% mean absolute percentage error) for dimers that are predominantly bound by electrostatic interactions, the comparatively larger error suggests that future work should 
focus on resolving the dichotomy between the local machine learning framework and the long-range nature of the intermolecular interactions.

\section{Acknowledgement}

The National Centre of Competence in Research (NCCR) "Materials' Revolution: Computational Design and Discovery of Novel Materials (MARVEL)" of the Swiss National Science Foundation (SNSF) and the EPFL are acknowledged for financial support. AG acknowledges funding from the Max Planck-EPFL center.

\section{Supporting Information Available}

The Supporting Information

\section{References}

(1) Stone, A. International Series of Monographs on Chemistry; Oxford University Press, 2013.

(2) Buckingham, A. D.; Fowler, P. W.; Hutson, J. M. Theoretical studies of van der Waals molecules and intermolecular forces. Chem. Rev. 1988, 88, 963-988.

(3) Castleman, Jr., A.; Hobza, P. van der Waais Molecules II: Introduction. Chem. Rev. 1994, 94, 1721-1722.

(4) Brutschy, B.; Hobza, P. van der Waals Molecules III: Introduction. Chem. Rev. 2000, 100, 3861-3862.

(5) Hobza, P.; Rezáč, J. Introduction: Noncovalent Interactions. Chem. Rev. 2016, 116, 4911-4912. 
(6) Pastorczak, E.; Corminboeuf, C. Perspective: Found in translation: Quantum chemical tools for grasping non-covalent interactions. J. Chem. Phys. 2017, 146, 120901.

(7) Parr, R.; Weitao, Y. Density-Functional Theory of Atoms and Molecules; International Series of Monographs on Chemistry; Oxford University Press, 1994.

(8) Kohn, W.; Sham, L. J. Self-Consistent Equations Including Exchange and Correlation Effects. Phys. Rev. 1965, 140, A1133-A1138.

(9) Cohen, A. J.; Mori-Sánchez, P.; Yang, W. Challenges for Density Functional Theory. Chem. Rev. 2012, 112, 289-320.

(10) Becke, A. D. Perspective: Fifty years of density-functional theory in chemical physics. J. Chem. Phys. 2014, 140, 18A301.

(11) Mardirossian, N.; Head-Gordon, M. Thirty years of density functional theory in computational chemistry: an overview and extensive assessment of 200 density functionals. Mol. Phys. 2017, 115, 2315-2372.

(12) Porezag, D.; Pederson, M. R. Infrared intensities and Raman-scattering activities within density-functional theory. Physical Review B 1996, 54, 7830-7836.

(13) Gilson, M. K.; Honig, B. H. Calculation of electrostatic potentials in an enzyme active site. Nature 1987, 330, 84-86.

(14) Mecozzi, S.; West, A. P.; Dougherty, D. A. Cation-pi interactions in aromatics of biological and medicinal interest: electrostatic potential surfaces as a useful qualitative guide. Proc. Nati. Acad. Sci. USA 1996, 93, 10566-10571.

(15) Sagara, T.; Klassen, J.; Ganz, E. Computational study of hydrogen binding by metalorganic framework-5. J. Chem. Phys. 2004, 121, 12543.

(16) Cardamone, S.; Hughes, T. J.; Popelier, P. L. A. Multipolar electrostatics. Phys. Chem. Chem. Phys. 2014, 16, 10367. 
(17) Polavarapu, P. L. Ab initio vibrational Raman and Raman optical activity spectra. J. Phys. Chem. 1990, 94, 8106-8112.

(18) Hughes, J. L. P.; Sipe, J. E. Calculation of second-order optical response in semiconductors. Phys. Rev. B 1996, 53, 10751-10763.

(19) Sipe, J. E.; Shkrebtii, A. I. Second-order optical response in semiconductors. Phys. Rev. B 2000, 61, 5337-5352.

(20) Sharma, S.; Ambrosch-Draxl, C. Second-Harmonic Optical Response from First Principles. Phys. Scr. 2004, T109, 128.

(21) Masunov, A. E.; Tannu, A.; Dyakov, A. A.; Matveeva, A. D.; Freidzon, A. Y.; Odinokov, A. V.; Bagaturyants, A. A. First principles crystal engineering of nonlinear optical materials. I. Prototypical case of urea. J. Chem. Phys. 2017, 146, 244104.

(22) Koritsanszky, T. S.; Coppens, P. Chemical Applications of X-ray Charge-Density Analysis. Chem. Rev. 2001, 101, 1583-1628.

(23) Lecomte, C.; Guillot, B.; Muzet, N.; Pichon-Pesme, V.; Jelsch, C. Ultra-high-resolution X-ray structure of proteins. Cell. Mol. Life Sci. 2004, 61, 774-782.

(24) Jayatilaka, D.; Dittrich, B. X-ray structure refinement using aspherical atomic density functions obtained from quantum-mechanical calculations. Acta Crystallogr. A 2008, $64,383-393$.

(25) Schnieders, M. J.; Fenn, T. D.; Pande, V. S.; Brunger, A. T. Polarizable atomic multipole X-ray refinement: application to peptide crystals. Acta Crystallogr. D 2009, 65, $952-965$.

(26) Brunger, A.; Adams, P. Comprehensive Biophysics; Elsevier, 2012; pp 105-115.

(27) Gatti, C.; Macchi, P. In Modern Charge-Density Analysis; Gatti, C., Macchi, P., Eds.; Springer Netherlands: Dordrecht, 2012. 
(28) Bader, R. F. W. A quantum theory of molecular structure and its applications. Chem. Rev. 1991, 91, 893-928.

(29) Bader, R. In Oxford University Press; Matta, C. F., Boyd, R. J., Eds.; Wiley-VCH Verlag GmbH \& Co. KGaA: Weinheim, Germany, 2007.

(30) de Silva, P.; Corminboeuf, C. Simultaneous Visualization of Covalent and Noncovalent Interactions Using Regions of Density Overlap. J. Chem. Theory Comput. 2014, 10, $3745-3756$.

(31) Johnson, E. R.; Keinan, S.; Mori-Sanchez, P.; Contreras-Garcia, J.; Cohen, A. J.; Yang, W. Revealing Noncovalent Interactions. J. Am. Chem. Soc 2010, 132, 64986506.

(32) Contreras-García, J.; Johnson, E. R.; Keinan, S.; Chaudret, R.; Piquemal, J.-P.; Beratan, D. N.; Yang, W. NCIPLOT: A Program for Plotting Noncovalent Interaction Regions. J. Chem. Theory Comput. 2011, 7, 625-632.

(33) Walker, P. D.; Mezey, P. G. Molecular electron density Lego approach to molecule building. J. Am. Ceram. Soc. 1993, 115, 12423-12430.

(34) Walker, P. D.; Mezey, P. G. Ab Initio Quality Electron Densities for Proteins: A MEDLA Approach. J. Am. Chem. Soc. 1994, 116, 12022-12032.

(35) Exner, T. E.; Mezey, P. G. Ab Initio-Quality Electrostatic Potentials for Proteins: An Application of the ADMA Approach. J. Phys. Chem. A 2002, 106, 11791-11800.

(36) Exner, T. E.; Mezey, P. G. Ab initio quality properties for macromolecules using the ADMA approach. J. Comput. Chem. 2003, 24, 1980-1986.

(37) Szekeres, Z.; Exner, T.; Mezey, P. G. Fuzzy fragment selection strategies, basis set dependence and HF-DFT comparisons in the applications of the ADMA method of macromolecular quantum chemistry. Int. J. Quantum Chem. 2005, 104, 847-860. 
(38) Stoll, H.; Wagenblast, G.; Preu $\beta$, H. On the use of local basis sets for localized molecular orbitals. Theor. Chim. Acta 1980, 57, 169-178.

(39) Meyer, B.; Guillot, B.; Ruiz-Lopez, M. F.; Genoni, A. Libraries of Extremely Localized Molecular Orbitals. 1. Model Molecules Approximation and Molecular Orbitals Transferability. J. Chem. Theory Comput. 2016, 12, 1052-1067.

(40) Meyer, B.; Guillot, B.; Ruiz-Lopez, M. F.; Jelsch, C.; Genoni, A. Libraries of Extremely Localized Molecular Orbitals. 2. Comparison with the Pseudoatoms Transferability. J. Chem. Theory Comput. 2016, 12, 1068-1081.

(41) Meyer, B.; Genoni, A. Libraries of Extremely Localized Molecular Orbitals. 3. Construction and Preliminary Assessment of the New Databanks. J. Phys. Chem. A 2018,

(42) Hirshfeld, F. L. Difference densities by least-squares refinement: fumaramic acid. Acta Crystallogr. B 1971, 27, 769-781.

(43) Stewart, R. F. Electron population analysis with rigid pseudoatoms. Acta Crystallogr. A 1976, 32, 565-574.

(44) Hansen, N. K.; Coppens, P. Testing aspherical atom refinements on small-molecule data sets. Acta Crystallogr. A 1978, 34, 909-921.

(45) Pichon-Pesme, V.; Lecomte, C.; Lachekar, H. On Building a Data Bank of Transferable Experimental Electron Density Parameters Applicable to Polypeptides. The Journal of Physical Chemistry 1995, 99, 6242-6250.

(46) Jelsch, C.; Pichon-Pesme, V.; Lecomte, C.; Aubry, A. Transferability of multipole charge-density parameters: application to very high resolution oligopeptide and protein structures. Acta Crystallogr., Sect. D 1998, 54, 1306-1318.

(47) Zarychta, B.; Pichon-Pesme, V.; Guillot, B.; Lecomte, C.; Jelsch, C. On the application 
of an experimental multipolar pseudo-atom library for accurate refinement of smallmolecule and protein crystal structures. Acta Crystallogr., Sect. A 2007, 63, 108-125.

(48) Lecomte, C.; Jelsch, C.; Guillot, B.; Fournier, B.; Lagoutte, A. Ultrahigh-resolution crystallography and related electron density and electrostatic properties in proteins. Journal of Synchrotron Radiation 2008, 15, 202-203.

(49) Domagala, S.; Munshi, P.; Ahmed, M.; Guillot, B.; Jelsch, C. Structural analysis and multipole modelling of quercetin monohydrate a quantitative and comparative study. Acta Crystallogr., Sect. B 2011, 67, 63-78.

(50) Domagala, S.; Fournier, B.; Liebschner, D.; Guillot, B.; Jelsch, C. An improved experimental databank of transferable multipolar atom models ELMAM2. Construction details and applications. Acta Crystallogr., Sect. A 2012, 68, 337-351.

(51) Koritsanszky, T.; Volkov, A.; Coppens, P. Aspherical-atom scattering factors from molecular wave functions. 1. Transferability and conformation dependence of atomic electron densities of peptides within the multipole formalism. Acta Crystallogr., Sect. A 2002, 58, 464-472.

(52) Dominiak, P. M.; Volkov, A.; Li, X.; Messerschmidt, M.; Coppens, P. A Theoretical Databank of Transferable Aspherical Atoms and Its Application to Electrostatic Interaction Energy Calculations of Macromolecules. J. Chem. Theory Comput. 2007, 3, $232-247$.

(53) Dittrich, B.; Koritsnszky, T.; Luger, P. A Simple Approach to Nonspherical Electron Densities by Using Invarioms. Angew. Chem., Int. Ed. 2004, 43, 2718-2721.

(54) Hathwar, V. R.; Thakur, T. S.; Row, T. N. G.; Desiraju, G. R. Transferability of Multipole Charge Density Parameters for Supramolecular Synthons: A New Tool for Quantitative Crystal Engineering. Cryst. Growth Des. 2011, 11, 616-623. 
(55) Brockherde, F.; Vogt, L.; Li, L.; Tuckerman, M. E.; Burke, K.; Müller, K.-R. Bypassing the Kohn-Sham equations with machine learning. Nature Communications 2017, 8, 872 .

(56) Bogojeski, M.; Brockherde, F.; Vogt-Maranto, L.; Li, L.; Tuckerman, M. E.; Burke, K.; Müller, K.-R. Efficient prediction of 3D electron densities using machine learning. arXiv:1811.06255 2018,

(57) Grisafi, A.; Wilkins, D. M.; Csányi, G.; Ceriotti, M. Symmetry-Adapted Machine Learning for Tensorial Properties of Atomistic Systems. Phys. Rev. Lett. 2018, 120, 036002.

(58) Grisafi, A.; Fabrizio, A.; Meyer, B.; Wilkins, D. M.; Corminboeuf, C.; Ceriotti, M. Transferable Machine-Learning Model of the Electron Density. ACS Centr. Sci. 2019, $5,57-64$.

(59) Alred, J. M.; Bets, K. V.; Xie, Y.; Yakobson, B. I. Machine learning electron density in sulfur crosslinked carbon nanotubes. Compos. Sci. Technol. 2018, 166, 3-9.

(60) Chandrasekaran, A.; Kamal, D.; Batra, R.; Kim, C.; Chen, L.; Ramprasad, R. Solving the electronic structure problem with machine learning. npj Comput. Mater. 2019, 5, 22.

(61) Fowler, A. T.; Pickard, C. J.; Elliott, J. A. Managing uncertainty in data-derived densities to accelerate density functional theory. J. Phys. Mater. 2019, 2, 034001.

(62) Jurečka, P.; Šponer, J.; Černý, J.; Hobza, P. Benchmark database of accurate (MP2 and $\operatorname{CCSD}(\mathrm{T})$ complete basis set limit) interaction energies of small model complexes, DNA base pairs, and amino acid pairs. Phys. Chem. Chem. Phys. 2006, 8, 1985-1993.

(63) Zhao, Y.; Truhlar, D. G. Density Functionals with Broad Applicability in Chemistry. Acc. Chem. Res. 2008, 41, 157-167. 
(64) Řzeáč, J.; Riley, K. E.; Hobza, P. S66: A well-balanced database of benchmark interaction energies relevant to biomolecular structures. J. Chem. Theory Comput. 2011, 7, $2427-2438$.

(65) Burns, L. A.; Vázquez-Mayagoitia, Á.; Sumpter, B. G.; Sherrill, C. D. Densityfunctional approaches to noncovalent interactions: A comparison of dispersion corrections (DFT-D), exchange-hole dipole moment (XDM) theory, and specialized functionals. J.Chem Phys. 2011, 134, 084107.

(66) Marshall, M. S.; Burns, L. A.; Sherrill, C. D. Basis set convergence of the coupled-cluster correction, MP2CCSD(T): Best practices for benchmarking non-covalent interactions and the attendant revision of the S22, NBC10, HBC6, and HSG databases. J. Chem. Phys. 2011, 135, 194102.

(67) Risthaus, T.; Grimme, S. Benchmarking of London Dispersion-Accounting Density Functional Theory Methods on Very Large Molecular Complexes. Journal of chemical theory and computation 2013, 9, 1580-91.

(68) Schneebeli, S. T.; Bochevarov, A. D.; Friesner, R. A. Parameterization of a B3LYP specific correction for noncovalent interactions and basis set superposition error on a gigantic data set of $\operatorname{CCSD}(\mathrm{T})$ quality noncovalent interaction energies. J. Chem. Theory Comput. 2011, 7, 658-668.

(69) Mardirossian, N.; Head-Gordon, M. $\omega$ B97M-V: A combinatorially optimized, rangeseparated hybrid, meta-GGA density functional with VV10 nonlocal correlation. J. Chem. Phys. 2016,

(70) Burns, L. A.; Faver, J. C.; Zheng, Z.; Marshall, M. S.; Smith, D. G.; Vanommeslaeghe, K.; MacKerell, A. D.; Merz, K. M.; Sherrill, C. D. The BioFragment Database (BFDb): An open-data platform for computational chemistry analysis of noncovalent interactions. J. Chem. Phys. 2017, 147, 161727. 
(71) Grisafi, A.; Wilkins, D. M.; Willatt, M. J.; Ceriotti, M. Atomic-scale representation and statistical learning of tensorial properties. arXiv:1904.01623 2019,

(72) Helgaker, T.; Jørgensen, P.; Olsen, J. Molecular Electronic-Structure Theory; John Wiley \& Sons, Ltd: Chichester, UK, 2000.

(73) Whitten, J. L. Coulombic potential energy integrals and approximations. J. Chem. Phys. 1973, 58, 4496-4501.

(74) Dunlap, B. I.; Connolly, J. W. D.; Sabin, J. R. On the applicability of LCAO-X $\alpha$ methods to molecules containing transition metal atoms: The nickel atom and nickel hydride. Int. J. Quantum Chem. Symp. 1977, 11, 81-87.

(75) Feyereisen, M.; Fitzgerald, G.; Komornicki, A. Use of approximate integrals in ab initio theory. An application in MP2 energy calculations. Chem. Phys. Lett. 1993, 208, 359363.

(76) Rendell, A. P.; Lee, T. J. Coupledcluster theory employing approximate integrals: An approach to avoid the input/output and storage bottlenecks. J. Chem. Phys. 1994, 101, 400-408.

(77) Eichkorn, K.; Treutler, O.; Öhm, H.; Häser, M.; Ahlrichs, R. Auxiliary basis sets to approximate Coulomb potentials. Chem. Phys. Lett. 1995, 240, 283-290.

(78) Weigend, F. A fully direct RI-HF algorithm: Implementation, optimised auxiliary basis sets, demonstration of accuracy and efficiency. Phys. Chem. Chem. Phys. 2002, 4, $4285-4291$.

(79) Werner, H.-J.; Manby, F. R.; Knowles, P. J. Fast linear scaling second-order MøllerPlesset perturbation theory (MP2) using local and density fitting approximations. $J$. Chem. Phys. 2003, 118, 8149-8160. 
(80) Kohn, W. Density Functional and Density Matrix Method Scaling Linearly with the Number of Atoms. Phys. Rev. Lett. 1996, 76, 3168-3171.

(81) Prodan, E.; Kohn, W. Nearsightedness of electronic matter. Proc. Nati. Acad. Sci. USA 2005, 102, 11635-11638.

(82) NAGY, A.; MARCH, N. H. Ratio of density gradient to electron density as a local wavenumber to characterize the ground state of spherical atoms. Mol. Phys. 1997, 90, $271-276$.

(83) Bohórquez, H. J.; Boyd, R. J. On the local representation of the electronic momentum operator in atomic systems. J. Chem. Phys. 2008, 129, 024110.

(84) Nagy, Á.; Liu, S. Local wave-vector, Shannon and Fisher information. Phys. Lett. A 2008, 372, 1654-1656.

(85) Murray, J. S.; Brinck, T.; Lane, P.; Paulsen, K.; Politzer, P. Statistically-based interaction indices derived from molecular surface electrostatic potentials: a general interaction properties function (GIPF). J. Mol. Struct. 1994, 30\%, 55-64.

(86) Murray, J. S.; Politzer, P. Statistical analysis of the molecular surface electrostatic potential: an approach to describing noncovalent interactions in condensed phases. $J$. Mol. Struct. 1998, 425, 107-114.

(87) Bootsma, A. N.; Doney, A. C.; Wheeler, S. Predicting the Strength of Stacking Interactions Between Heterocycles and Aromatic Amino Acid Side Chains. 2019,

(88) Bootsma, A. N.; Wheeler, S. Converting SMILES to Stacking Interaction Energies. 2019 ,

(89) Volkov, A.; Koritsanszky, T.; Coppens, P. Combination of the exact potential and multipole methods (EP/MM) for evaluation of intermolecular electrostatic interaction 
energies with pseudoatom representation of molecular electron densities. Chem. Phys. Lett. 2004, 391, 170-175.

(90) Bootsma, A. N.; Wheeler, S. E. Tuning Stacking Interactions between AspArg Salt Bridges and Heterocyclic Drug Fragments. J. Chem. Inf. Model. 2019, 59, 149-158.

(91) Berman, H. M. The Protein Data Bank. Nucleic Acids Res. 2000, 28, 235-242. 


\title{
Insights into non-covalent interactions with a machine-learned electron density
}

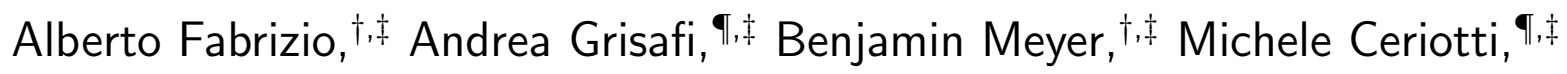 \\ and Clemence Corminboeuf*, $\uparrow, \ddagger$ \\ $\dagger$ †aboratory for Computational Molecular Design, Institute of Chemical Sciences and \\ Engineering,École Polytechnique Fédérale de Lausanne, CH-1015 Lausanne, Switzerland \\ $\ddagger$ National Centre for Computational Design and Discovery of Novel Materials (MARVEL), \\ École Polytechnique Fédérale de Lausanne, 1015 Lausanne, Switzerland \\ \Laboratory of Computational Science and Modeling, IMX, École Polytechnique Fédérale \\ de Lausanne, 1015 Lausanne, Switzerland \\ E-mail: clemence.corminboeuf@epfl.ch
}




\begin{abstract}
Chemists continuously harvest the power of non-covalent interactions to control phenomena in both the micro- and macroscopic worlds. From the quantum chemical perspective, the strategies essentially rely upon an in-depth understanding of the physical origin of these interactions, the quantification of their magnitude and their visualization in real-space. The total electron density $\rho(\boldsymbol{r})$ represents the simplest yet most comprehensive piece of information available for fully characterizing bonding patterns and non-covalent interactions. The charge density of a molecule can be computed by solving the Schrödinger equation, but this approach becomes rapidly demanding if the electron density has to be evaluated for thousands of different molecules or for very large chemical systems, such as peptides and proteins. Here we present a transferable and scalable machine-learning model capable of predicting the total electron density directly from the atomic coordinates. The regression model is used to access qualitative and quantitative insights beyond the underlying $\rho(\boldsymbol{r})$ in a diverse ensemble of sidechain-sidechain dimers extracted from the BioFragment database (BFDb). The transferability of the model to more complex chemical systems is demonstrated by predicting and analyzing the electron density of a collection of 8 polypeptides.
\end{abstract}

\title{
Graphical TOC Entry
}

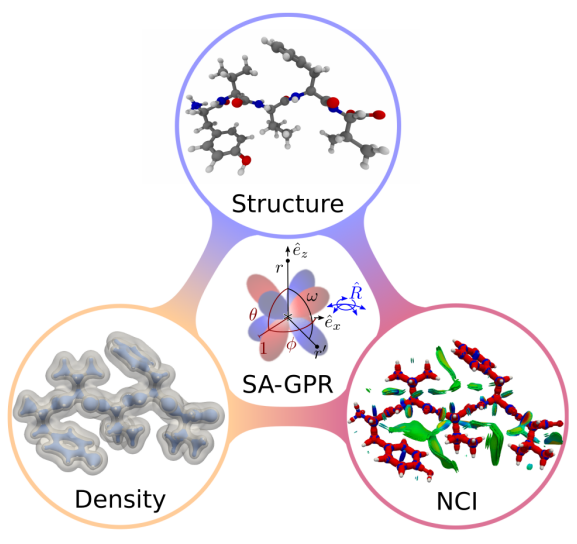




\section{Introduction}

Non-covalent interactions (NCIs) govern a multitude of chemical phenomena and are key components for constructing molecular architectures. ${ }^{1}$ Their importance fostered an intense research effort to accurately quantify their magnitude and develop an intuitive characterization of their physical nature using quantum chemistry. ${ }^{2-6}$ Among the different approaches to characterize non-covalent interactions, one of the simplest and most generally applicable takes as a starting point the electron density $\rho(\boldsymbol{r})$ that encodes, in principle, all the information needed to fully characterize a chemical system. ${ }^{7}$ Despite the fact that the universal functional relationship between total energy and $\rho(\boldsymbol{r})$ remains unknown, existing approximations within the framework of Kohn-Sham DFT $(\mathrm{KS}-\mathrm{DFT})^{8}$ do permit access to all molecular properties within a reasonable degree of accuracy. ${ }^{9-11}$

Properties that can be derived exactly from the electron density distribution include molecular and atomic electrostatic moments (e.g., charges, dipole, quadrupoles), electrostatic potentials and electrostatic interaction energies. Knowledge of these quantities is fundamental in diverse chemical applications, including the computation of the IR intensities, ${ }^{12}$ the identification of binding sites in host-guest compounds, ${ }^{13-15}$ and the exact treatment of electrostatics within molecular simulations. ${ }^{16}$ Moreover, analyzing the deformation of $\rho(\boldsymbol{r})$ in the presence of an external field provides access to another set of fundamental properties, namely molecular static (hyper)polarizabilities and, thus, to the computation of Raman spectra $^{17}$ and non-linear optical properties. ${ }^{18-21}$

The natural representation of the electron density in real space makes it especially suitable for accessing spatial information about structural and electronic molecular properties, including X-Ray structure refinement ${ }^{22-27}$ and representations using scalar fields. ${ }^{6}$ Routinely

used examples include the quantum theory of atoms in molecules (QTAIM), ${ }^{28,29}$ the density overlap region indicator (DORI), ${ }^{30}$ and the non-covalent interaction (NCI) index. ${ }^{31,32}$

$\rho(\boldsymbol{r})$ is generally obtained by solving the electronic structure problem through ab-initio computations. The main advantage of this approach is that it returns the variationally op- 
timized electronic density for a given Hamiltonian. Yet, ab-initio computations can become increasingly burdensome if $\rho(\boldsymbol{r})$ has to be evaluated for thousands of different molecules or for very large chemical systems, such as peptides and proteins. These large scale problems are typically tackled using a more scalable approach that consists of either using linear scaling techniques such as Mezey's molecular electron density LEGO assembler (MEDLA) ${ }^{33,34}$ and adjustable density matrix assembler (ADMA), ${ }^{35-37}$ as well as approaches based on localized molecular orbitals, such as ELMO. ${ }^{38-41}$ Another methodology belonging to this second category involves the use of experimental techniques, such as X-Ray diffraction, to probe the electron density and subsequently reconstructing $\rho(\boldsymbol{r})$ through multipolar models ${ }^{42-44}$ and pseudo-atomic libraries, such as ELMAM, ${ }^{45-48}$ ELMAM2,${ }^{49,50}$ UBDB, ${ }^{51,52}$ Invarioms $^{53}$ and SBFA. ${ }^{54}$ While successful, these two methodologies have intrinsic limits: the first is unable to capture the deformations of the charge density due to intermolecular interactions unless a suitable fragment is generated $a d-h o c$, while the second relies on experimental data and is difficult to extend to thousands of different chemical systems at once. Recently, the development of several machine-learning models targeting the electron density has effectively established a third promising methodology, with the potential to overcome the limitations of the more traditional approaches.

The first machine-learning model of $\rho(\boldsymbol{r})$ was developed on the basis of the HohenbergKohn mapping between the nuclear potential and the electron density. ${ }^{55,56}$ Although successful, the choice of the nuclear potential as a representation of the different molecular conformations and the expansion of the electron density in an orthogonal plane-wave basis effectively constrained this landmark model to relatively small and rigid molecules with limited transferability to larger systems. Recently, we proposed an atom-centered, symmetryadapted Gaussian process regression ${ }^{57}$ (SA-GPR) framework explicitly targeting the learning of the electron density. ${ }^{58}$ Using an optimized non-orthogonal basis set, pseudo-valence electron densities could be predicted in a linear-scaling and transferable manner, meaning that the model is able to tackle much larger chemical systems than the those used to train the 
regression model. A third approach, that can also achieve transferability between different systems, uses a direct grid-based representation of the atomic environment to learn and predict the electronic density in each point of the molecular space. ${ }^{59-61}$ Representing the density field on a large set of grids points rather than on a basis set effectively avoids the introduction of a basis set error, but also dramatically increases the computational effort.

One should also consider that machine learning, being a data-driven approach, requires high-quality, diverse reference data. Fortunately, several specialized benchmark databases that target NCIs have appeared over the past decade. From the original S22 ${ }^{62}$ to NCIE53, ${ }^{63}$ S66, ${ }^{64}$ NBC10ext ${ }^{65,66}$ and S12L,${ }^{67}$ the evolution of these datasets has, generally, followed a prescription of increasing the number of entries, principally by including subtler interactions and/or larger systems. In this respect, the databases of Friesner, ${ }^{68}$ Head-Gordon ${ }^{69}$ and the recent $\mathrm{BFDb}$ of Sherrill, ${ }^{70}$ constitute a special category because of their exceptional size (reaching thousands of entries) which are now sufficiently large to be compatible with machine-learning applications. Beyond their conceptual differences, each of these benchmark sets aim at improving the capability of electronic structure methods to describe the energetic aspects of non-covalent interactions.

In this work, we introduce a dramatic improvement of our previous density-learning approach by making the regression machinery of $\rho(\boldsymbol{r})$ compatible with density-fitting auxiliary basis sets. These specialized basis sets are routinely used in quantum chemistry to approximate two-center one-electron densities. Here, the auxiliary basis sets are used directly to represent the electron densities that enter our machine-learning model, with the additional advantage of avoiding the arbitrary basis set optimization procedures on the machine-learning side. This enhanced framework leverages the transferability of our symmetry-adapted regression method and is capable of learning the all-electron density across a vast spectrum of 2291 chemically diverse dimers formed by sidechain-sidechain interactions extracted from the BioFragment Database (BFDb). ${ }^{70}$ The performance of the method is demonstrated through the reproduction of $\rho(\boldsymbol{r})$ between and within each monomer forming the dimers. The ac- 
curacy of the predicted densities is assessed by computing density-based scalar fields and electrostatic potentials, while the errors made with respect to the reference densities are computed by direct integration on three-dimensional grids. As a major breakthrough, the model is used to predict the charge density of a set of 8 polypeptides $(\sim 100$ atoms $)$ at DFT accuracy in few minutes.

\section{Methods}

Gaussian process regression (GPR) can be extended to encode all the fundamental symmetries of the $O(3)$ group, effectively allowing machine-learning of all the molecular properties that transform as spherical tensors under rotation and inversion operations. ${ }^{57,71}$ In the specific case of the electron density, the scheme relies upon the decomposition of the field into additive, atom-centered contributions and the subsequent prediction of the corresponding expansion coefficients. ${ }^{58}$ In SA-GPR, each molecule is represented as a collection of atom-centered environments, whose relationships and similarities are measured by symmetry adapted kernels. An in-depth discussion about how a symmetry adapted regression model of the electron density can be constructed is reported in the supplementary materials.

The decomposition of the electron density in continuous atom-centered basis functions is the cornerstone of the scalability and transferability of our SA-GPR model. Beside being generally desirable, these properties are actually crucial to accurately describe the chemical diversity present in the BioFragment Database within a reasonable computational cost. On the other hand, the projection of the density field onto a basis set leads to an additional error on top of that which can be ascribed to machine learning. In practice, all the efforts placed into achieving a negligible machine-learning error are futile if the overall accuracy of the model is dictated by a large basis set decomposition error.

Standard quantum chemical basis sets are generally optimized to closely reproduce the behavior of atomic orbitals ${ }^{72}$ and results in unacceptable errors if used to decompose the 
electronic density (Figure 1). In contrast, specialized basis sets used in the density fitting approximation (also known as resolution-of-the-identity (RI) approximation) ${ }^{73-79}$ are specifically optimized to represent a linear expansion of one-electron charge densities obtained from the product of atomic orbitals. Using the RI-auxiliary basis sets $\left\{\phi_{k}^{R I}\right\}$, the total electron density field can be expressed as:

$$
\rho(\boldsymbol{r})=\sum_{k}^{N_{a u x}}\left(\sum_{a b}^{N_{A O}} D_{a b} d_{k}^{a b}\right) \phi_{k}^{R I}(\boldsymbol{r})=\sum_{k}^{N_{a u x}} c_{k} \phi_{k}^{R I}(\boldsymbol{r})
$$

where, $D_{a b}$ is the one-electron reduced density matrix and $d_{k}^{a b}$ are the RI-expansion coefficients.

Given a molecular geometry, the value of the basis functions can be readily computed at each point of space, leaving the $c_{k}$ expansion coefficients as the only ingredient needed to fully determine the amplitude of $\rho(\boldsymbol{r})$. In this sense, the prediction of the electron density by the SA-GPR model can be summarized as the prediction of the $c_{k}$ coefficients after training on a set of previously computed reference densities (details in the ESI).

As shown in Figure 1, the use of the RI-auxiliary basis sets results in nearly two orders of magnitude increase in the overall accuracy with respect to the corresponding standard basis set. The addition of diffuse functions marginally improves the performance of the decomposition, but leads to instabilities of the overlap matrix (high condition number) and increases dramatically the number of basis functions per atom. 

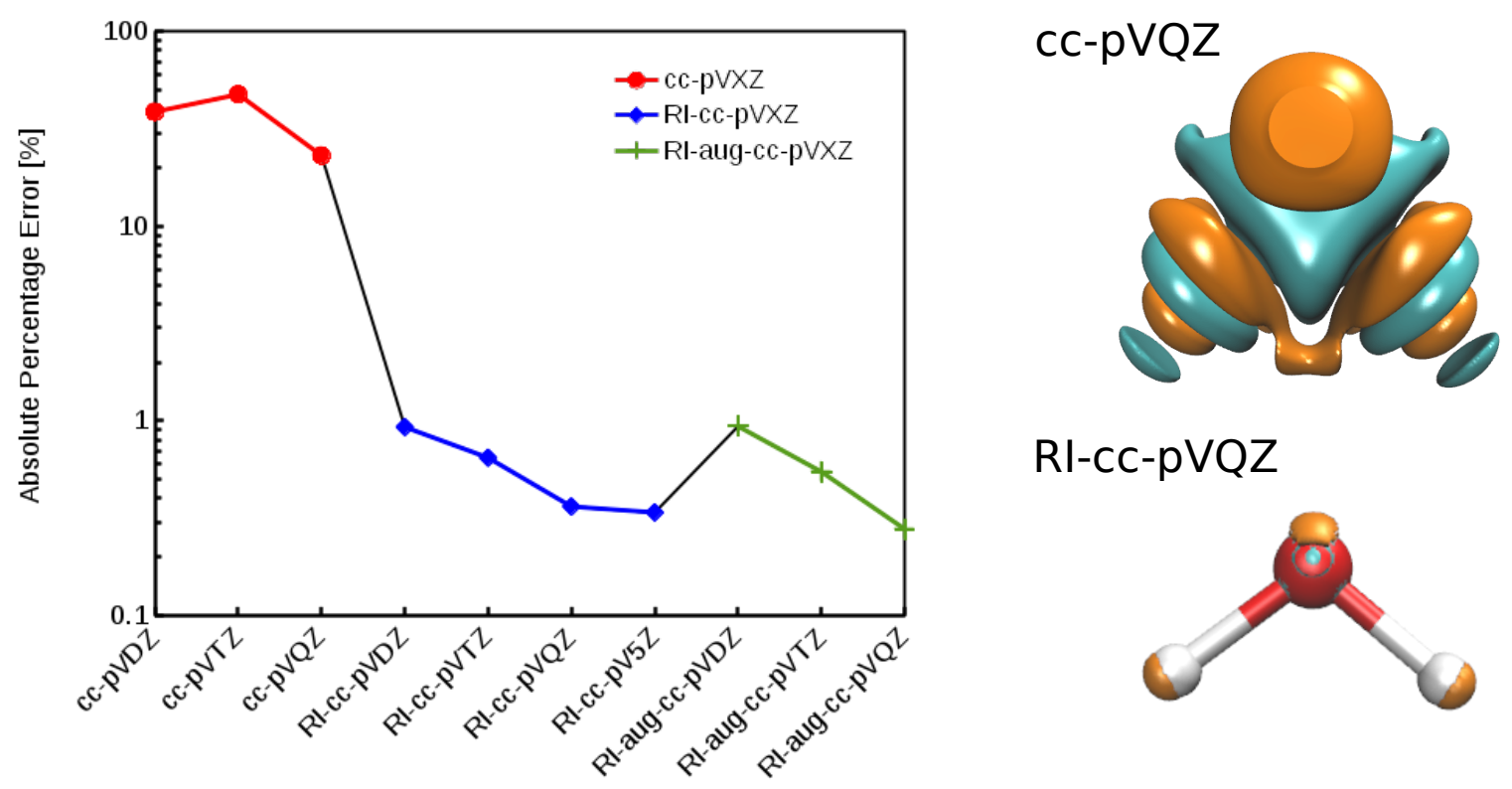

RI-cc-pVQZ

Figure 1: (left) Electron density of a single water molecule: evolution of the absolute percentage error depending on the choice of decomposition basis set. (right) Comparison of the density error made with the standard and the RI-auxiliary cc-pVQZ basis set ( cyan and orange isosurfaces refer to an error of $\pm 0.005 \mathrm{Bohr}^{-3}$ ). Reference density: PBE/cc-pVQZ.

In practice, Weigend's JK-fit cc-pVQZ ${ }^{78}$ basis set (henceforth: RI-cc-pVQZ) offers the best trade-off between accuracy and computational demand and therefore represents the best choice for the density decomposition.

\section{Computational Details}

The dataset of molecular dimers has been selected from the side-chain side-chain interaction (SSI) subset of the BioFragment Database (BFDb). ${ }^{70}$ The original set is made of 3558 dimers formed by amino-acids side-chain fragments taken from 47 different protein structures. Dimers with more than 25 atoms as well as those containing sulfur atoms were not considered. While the total number of sulfur-containing structures is too small to enable the machinelearning model to accurately capture its rich chemistry, the inclusion of the larger systems does not increase dramatically the chemical diversity of the dataset. The final dataset contains a total of 2291 dimers. 
As shown in Figure 2, the complete set of 2291 dimers spans a large variety of dominant interaction types, ranging from purely dispersion dominated complexes (in blue) to mixedinfluence (green and yellow) to hydrogen-bonded and charged systems (red). We retain the same classification criteria as in the original database to attribute the nature of the dominant interaction.

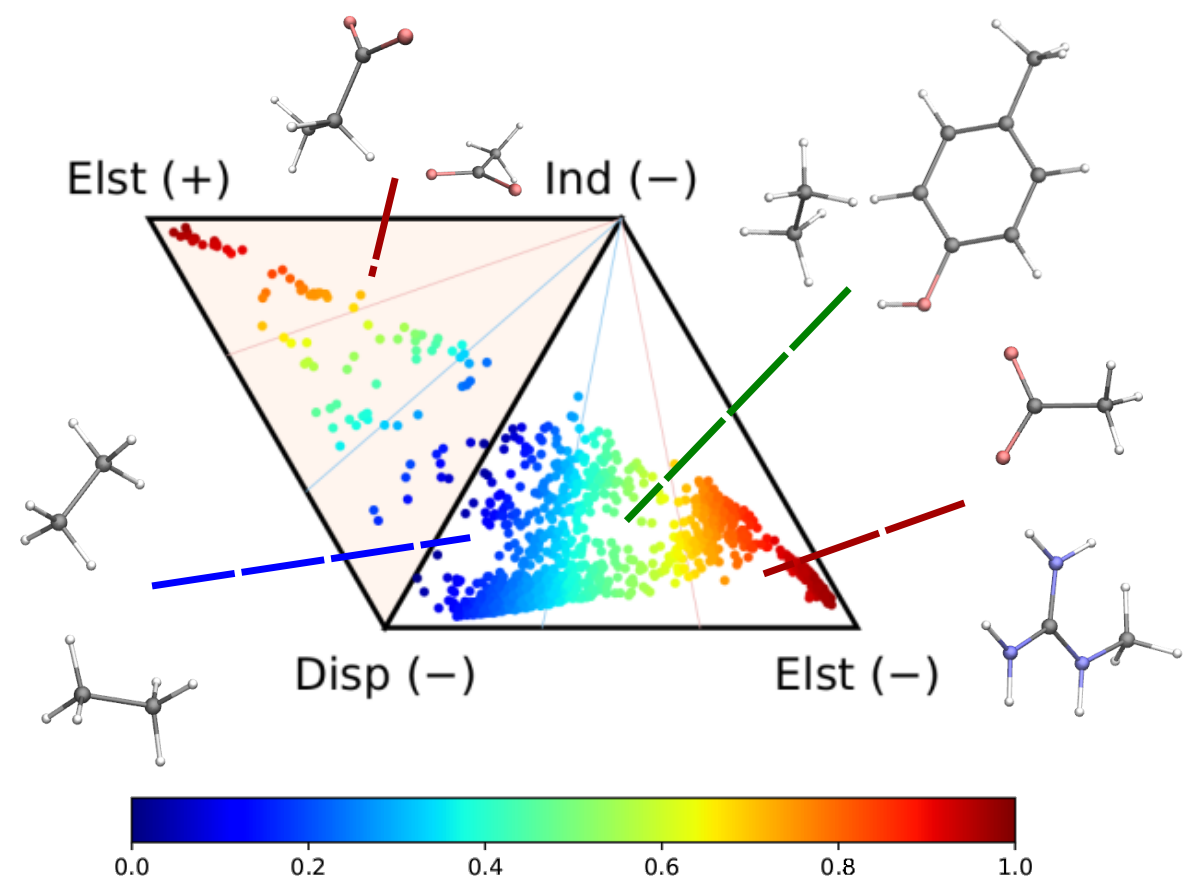

Figure 2: Ternary diagram representation of the attractive components of the dimer interaction energies for the 2291 systems considered in this work. The values of the SAPT analysis are taken from Ref. 70.

For each dimer, the reference full-electron density has been computed at the $\omega$ B97X-D/ccpVQZ level using the resolution of identity approximation for the Coulomb and exchange potential (RI-JK). This implies that RI-auxiliary functions up to $l=5$ are included for carbon, nitrogen and oxygen atoms while auxiliary functions up to $l=4$ are used for hydrogen atoms. 


\section{Results and Discussion}

The training set for the density-learning model was chosen by randomly picking 2000 dimers out of a total of 2291 possibilities. The remaining 291 were used to test the accuracy of the predictions. Given the tremendous number of possible atomic environments $(\sim 40000)$ associated with such a chemically diverse database, a subset of $M$ reference environments was selected to reduce the dimensionality of the regression problem (see Supplementary information). To assess the consequences of this dimensionality reduction, the learning exercise was performed on three different sizes $M=\{100,500,1000\}$ for the reference atomic environments. Figure 3 summarizes the performance of the machine learning algorithm, expressed in terms of the mean absolute difference between the predicted and ab-initio densities reconstructed on the RI auxiliary-basis, and weighted by the total number of electrons of each dimer.
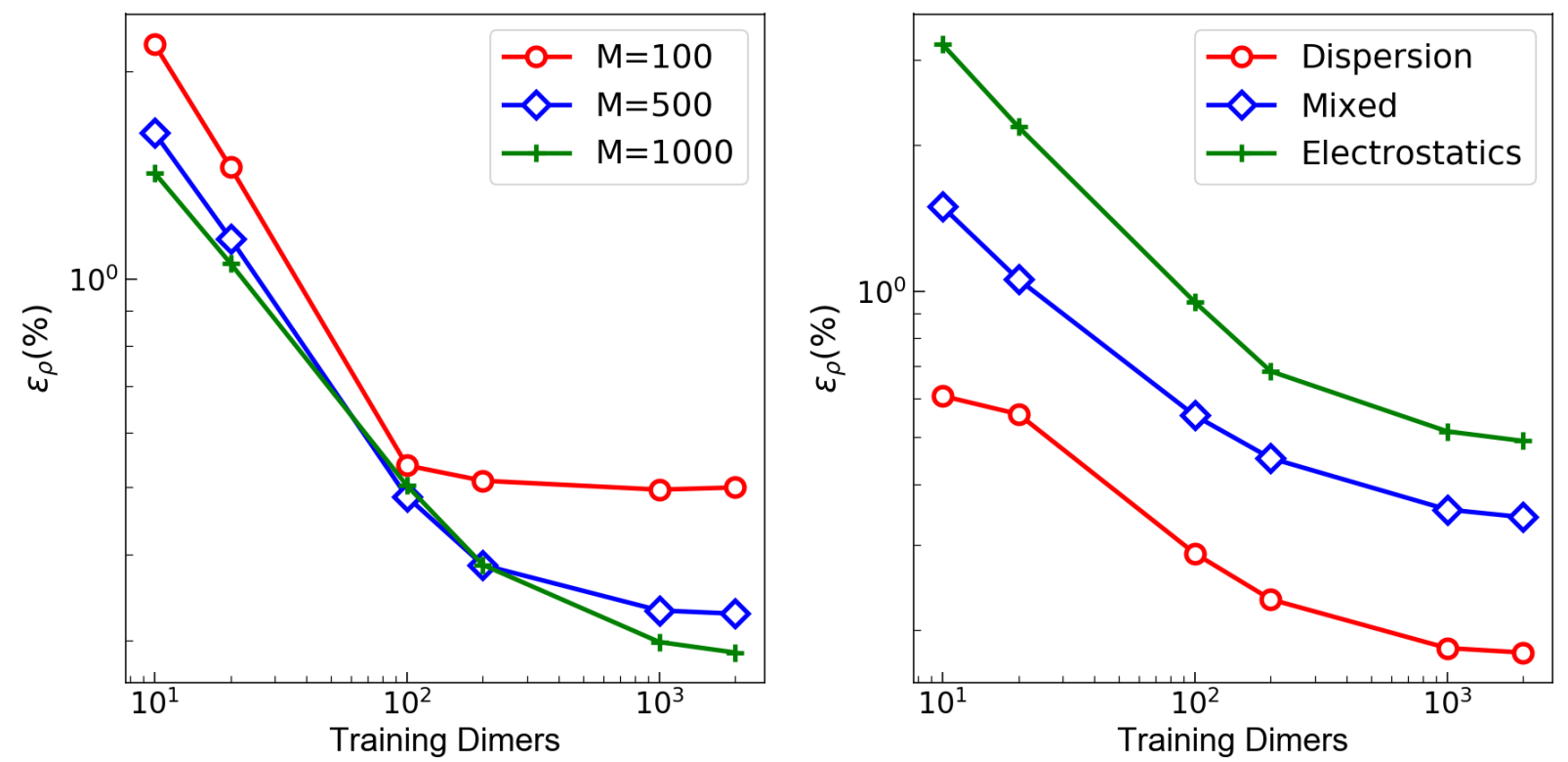

Figure 3: Learning curves. (left) weighted mean absolute percentage error $\left(\epsilon_{\rho}(\%)\right)$ of the predicted SA-GPR densities as a function of the number of training dimers. The weights correspond to the number of electrons in each dimer and the normalization is defined by the total number of electrons. Color code reflects the number of reference environments. (right) $\epsilon_{\rho}(\%)$ of the predicted SA-GPR densities $(\mathrm{M}=1000)$ divided per dominant contribution to the interaction energy according to Ref. [ 70]. $\epsilon_{\rho}(\%)=100 \times \frac{1}{N_{\mathrm{e}}} \sum_{i} N_{\mathrm{e}}^{i} \frac{\int d \boldsymbol{r}\left|\rho_{\mathrm{QM}}^{i}(\boldsymbol{r})-\rho_{\mathrm{ML}}^{i}(\boldsymbol{r})\right|}{\int d \boldsymbol{r} \rho_{\mathrm{QM}}^{i}(\boldsymbol{r})}$. 
As shown in the first panel of Figure 3, 100 training dimers were sufficient to reach saturation of the density error around $0.5 \%$ for $M=100$. This result already outperforms the level of accuracy reached in our previous work, which is remarkable given the large chemical diversity of the dataset and the consideration of all-electron densities. Learning curves obtained with $M=500$ and $M=1000$ show steeper slopes, approaching saturation at about 2000 training dimers with errors that were reduced to $\sim 0.2-0.3 \%$. The predicted full-electron densities are five times more accurate than the previous predictions of valence-only densities (approximately 1\%). ${ }^{58} \mathrm{~A}$ more detailed analysis of the $M=1000$ learning curve reveals a strong dependence on the nature of the dominant interaction (Figure 3). Specifically, stronger non-local character in the interaction yields a larger error. This is especially prevalent for dimers dominated by electrostatic interactions (i.e., hydrogen bonds, charged systems), which are characterized by errors that are twice as large as those found in other regimes.

The origin of this slow convergence arises from two factors. First, only about $20 \%$ of the dimers are dominantly bound by electrostatics. ${ }^{70}$ The priority of the regression model is thus to minimize the error on the other classes. Second, there is a fundamental dichotomy between the local nature of our symmetry-adapted learning scheme and the long-range nature of the interactions. In this respect, a global ML representation of the density field would be more suitable, but this would imply renouncing the scalability and transferability of the model. Given a large enough training set, however, our SA-GPR model is able to capture the density deformations due to the field generated by the neighboring molecule. The reason is rooted in the intrinsic locality of density deformations and in the concept of "nearsightedness" 80,81 of all local electronic properties, which constitutes a theoretical justification for a local decomposition of such quantities.

The fundamental advantage of setting the electron density as the machine-learning target is the broad spectrum of chemical properties that are directly derivable from $\rho(\boldsymbol{r})$. For instance, the predicted charge densities are the key ingredient in density-dependent scalar fields aimed at visualizing and characterizing interactions between atoms and molecules in 
real space. Examples of the density overlap region indicator (DORI) ${ }^{30}$ are given in Figure 4 for representative dimers. Compared to the rather featureless $\rho(\boldsymbol{r})$, DORI reveals fine details of electronic structure, which constitute a more sensitive probe for the quality of the machine-learning predictions. In particular, it reveals density overlaps (or clashes) associated with bonding and non-covalent regions on equal footing through the behavior of the local wave-vector $(\nabla \rho(\boldsymbol{r}) / \rho(\boldsymbol{r})) .{ }^{82-84}$

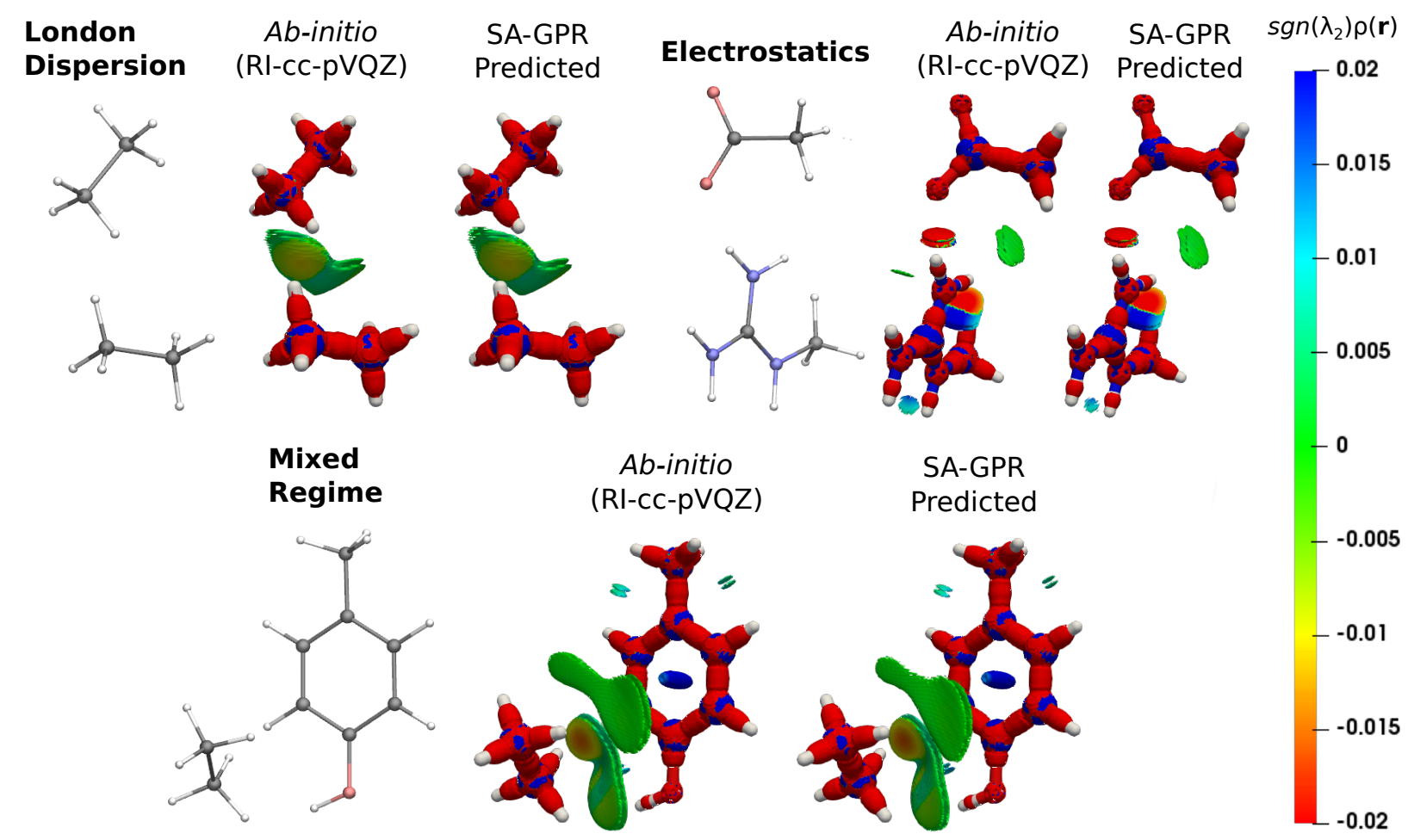

Figure 4: DORI maps of representative dimers for each type of dominant interaction (DORI isovalue: 0.9). Isosurfaces are color-coded ${ }^{31}$ with $\operatorname{sgn}\left(\lambda_{2}\right) \rho(\boldsymbol{r})$ in the range from attractive -0.02 a.u. (red) to repulsive 0.02 a.u. (blue). In particular, $\operatorname{sgn}\left(\lambda_{2}\right) \rho(\boldsymbol{r})<0$ characterizes covalent bonds or strongly attractive NCIs (e.g. H-bonds); $\operatorname{sgn}\left(\lambda_{2}\right) \rho(\boldsymbol{r}) \sim 0$ indicates weak attractive interactions (van der Waals); $\operatorname{sgn}\left(\lambda_{2}\right) \rho(\boldsymbol{r})>0$ repulsive NCIs (e.g. steric clashes).

As shown in Figure 4, the intra- and intermolecular DORI domains obtained with the SA-GPR densities are indistinguishable from those in the ab-initio maps. This performance is especially impressive for the density clashes associated with low density values, as is typical for the non-covalent domains. All the features are well captured by the predicted densities 
ranging from large and delocalized basins typical of the van der Waals complexes (in green) to the compact and directional domains typical of electrostatic interactions, to intramolecular steric clashes (e.g. phenol, mixed regime). Overall, these results illustrate that the residual $0.2 \%$ mean absolute percentage error does not significantly affect the density amplitude in the valence and intermolecular regions that are accurately described by the SA-GPR model. The highest amplitude errors are concentrated near the nuclei in the region dominated by the core-density fluctuations.

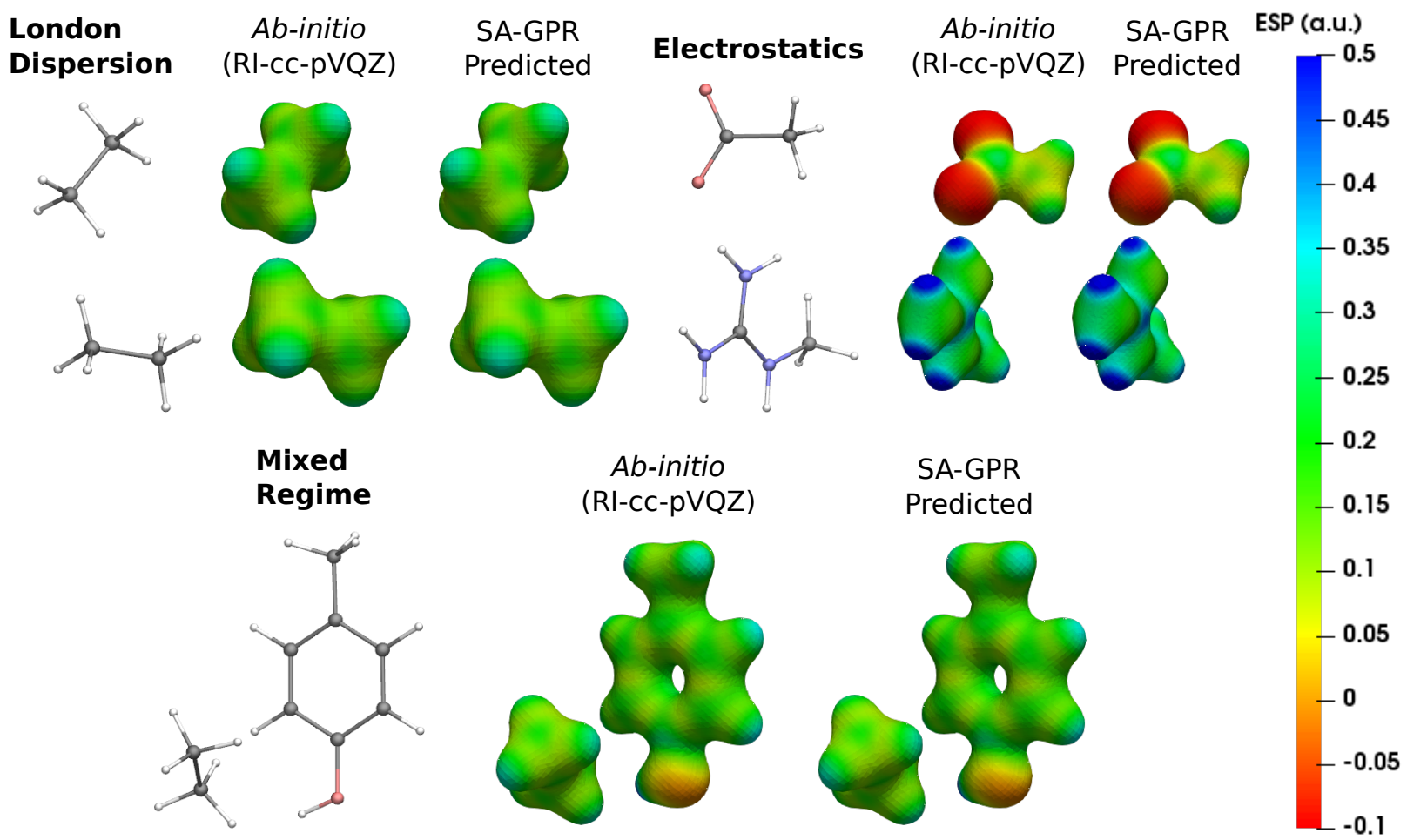

Figure 5: Electrostatic potential (ESP) maps of representative dimers for each type of dominant interaction (density isovalue: $0.05 \mathrm{e}^{-} \mathrm{Bohr}^{-3}$ ). ESP potential is given in Hartree atomic units (a.u.).

The versatility of the machine-learning prediction is further illustrated by using the predicted densities to compute the molecular electrostatic potential (ESP) for the same representative dimers (Figure 5). ESP maps based on predicted densities agree quantitatively with the ab-initio reference and correctly attribute the sign and magnitude of the electrostatic potential in all regions of space. Importantly, the accuracy of the ESP magnitude 
remains largely independent of the dominant interaction type. This is especially relevant for charged dimers (electrostatics) as it demonstrates that despite slower convergence of the learning curve for this category, the achieved accuracy of the model is sufficient to describe the key features of the electrostatic potential.

The most widespread applications of ESP maps exploit qualitative information (e.g., identification of the molecular regions most prone to electrophilic/nucleophilic attack) but the electrostatic potentials can be related to quantitative properties such as the degree of acidity of hydrogen bonds and the magnitude of binding energies. ${ }^{85-89}$ As a concrete example related to structure-based drug design, we used a recent model that estimates the strength of the stacking interactions between heterocycles and aromatic amino acid side-chains directly from the ESP maps. ${ }^{87,88,90}$ This model derives the stacking energies of drug-like heterocycles from the maximum and mean value of their ESP within a surface delimited by molecular van der Waals volume (at $3.25 \AA$ above the molecular plane). ${ }^{87}$ Following this procedure, we used the ESP derived from the ML predicted densities to compute the binding energies between a representative heterocycle included in our dataset, the tryptophan side-chain, and the three aromatic amino acid side-chains (Figure 6). 


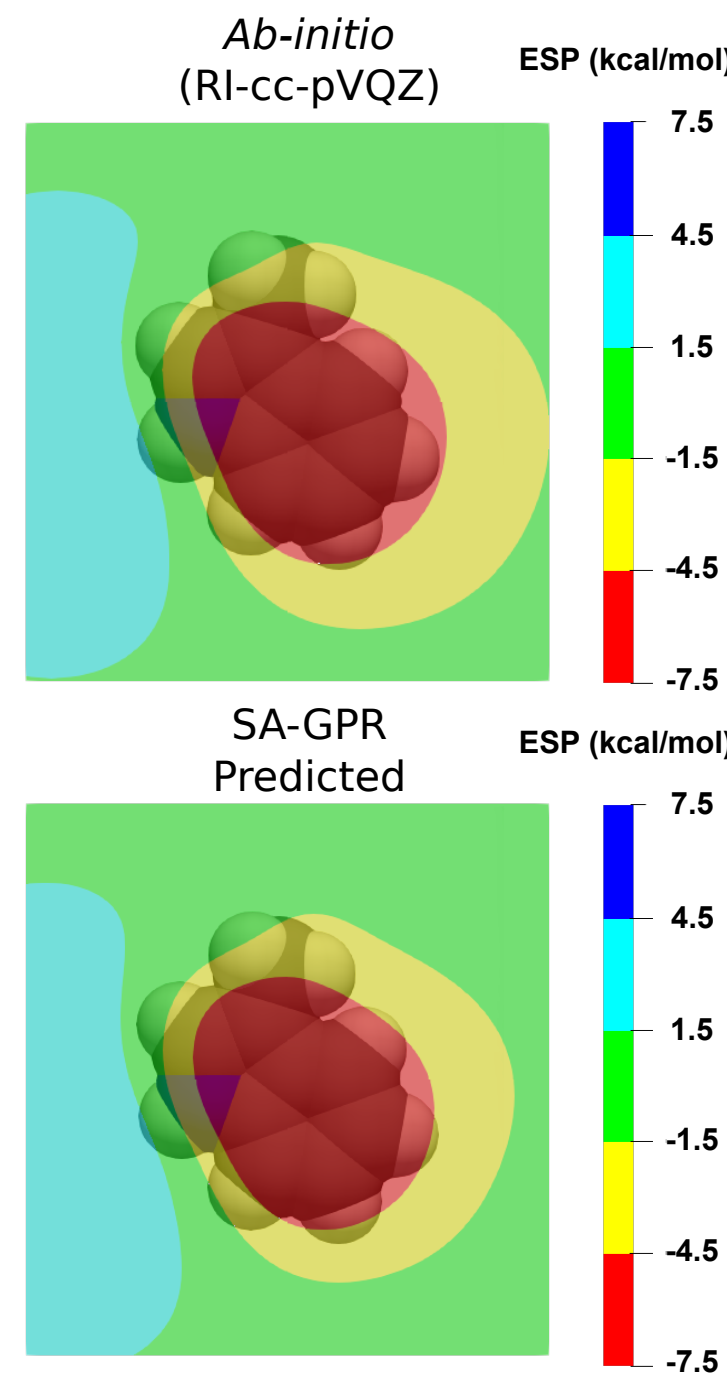

Ab-Initio Stacking Interaction Energy

PHE

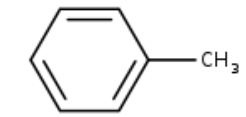

$-8.35 \mathrm{kcal} / \mathrm{mol}$

TYR

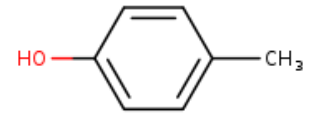

$-9.30 \mathrm{kcal} / \mathrm{mol}$

TRP<smiles>Cc1c[nH]c2ccccc12</smiles>

$-11.21 \mathrm{kcal} / \mathrm{mol}$

Predicted Stacking Interaction Energy

PHE

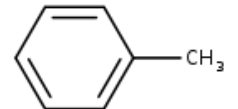

$-8.39 \mathrm{kcal} / \mathrm{mol}$

TYR

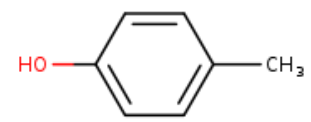

$-9.35 \mathrm{kcal} / \mathrm{mol}$

TRP<smiles>Cc1c[nH]c2ccccc12</smiles>

$-11.28 \mathrm{kcal} / \mathrm{mol}$

Figure 6: (left) Electrostatic potential maps $3.25 \AA$ above the plane of the tryptophan (TRP) side-chain. The van der Waals volume of TRP is represented in transparency. (Right) Stacking interaction energies of TRP with the phenylalanine (PHE), tyrosin (TYR) and tryptophan (TRP) side-chains computed as detailed in Ref. 87.

Comparison between $a b$-initio and ML predicted stacking interaction energies shows that the deviations in the ESP maps lead to minor errors on the order of $0.05 \mathrm{kcal} / \mathrm{mol}$. The largest deviations in the ESP would appear further away from the molecule, beyond the region exploited for the computation of the energy descriptors (i.e., the sum of the atomic van der Waals radii). This behavior is related to the propagation of the deviations in the density predictions $\delta \rho(\boldsymbol{r})$ to the electrostatic potential $\delta \hat{\phi}(\boldsymbol{k})=4 \pi \delta \hat{\rho}(\boldsymbol{k}) / k^{2}$. In particular, the error on the low frequency components of $\rho(\boldsymbol{r})$ is greatly enhanced as $k \rightarrow 0$, resulting 
in larger errors as the distance from the nuclei increases.

\section{Prediction on Polypeptides}

The tremendous advantage of the atom-centered density decomposition is to deliver a machinelearning model that depends only on the different atomic environments and not on the identity of the molecules included in the training set. Thanks to its transferability, the model provides access to density information of large macromolecules, at the sole price of including sufficient diversity, that can capture the chemical complexity of a larger system. The predictive power of this extrapolation procedure is demonstrated by using the machine-learning model exclusively trained on the $2291 \mathrm{BFDb}$ dimers to predict the electron density of 8 polypeptides taken from the Protein DataBank (PDB). ${ }^{91}$ The performance of the ML model for each macromolecules, labelled by their PBD ID, is reported in Figure 7.

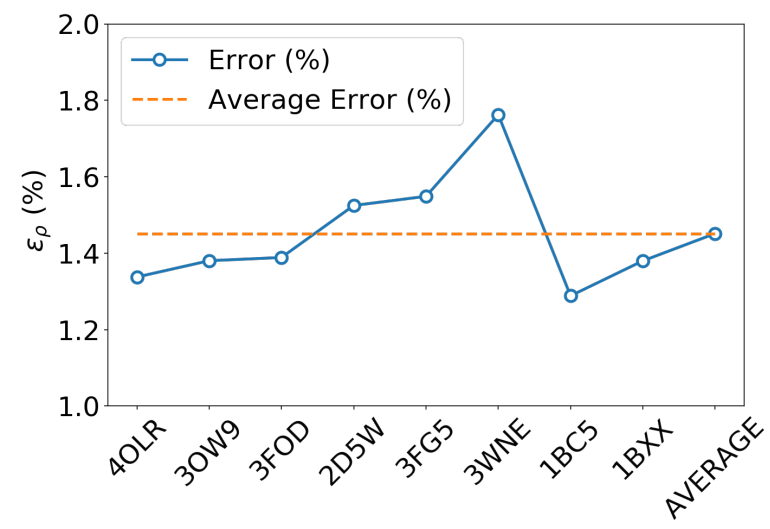

Figure 7: Weighted mean absolute percentage error $\left(\epsilon_{\rho}(\%)\right)$ of the predicted densities extrapolated for 8 biologically relevant peptides (protein databank ID).

Overall, the predictions lead to a low average error of only $1.5 \%$ for the 8 polypeptides, which is in line with the highest density errors obtained on the BFDb test set. Relevantly, the largest discrepancies are obtained for 3WNE, which is the only cyclopeptide of the set. The origin of these differences can be understood by performing a more detailed analysis on a representative polypeptide, the leu-enkephalin (4OLR). The errors in this percentage range 
do not affect the density-based properties, such as the spatial analysis of the intramolecular interactions with scalar fields (Figure 8 top right panel). Yet, most of the discrepancies occur along the amino acid backbone (Figure 8 lower panels), which is especially sensitive for the more strained 3WNE cyclopeptide.
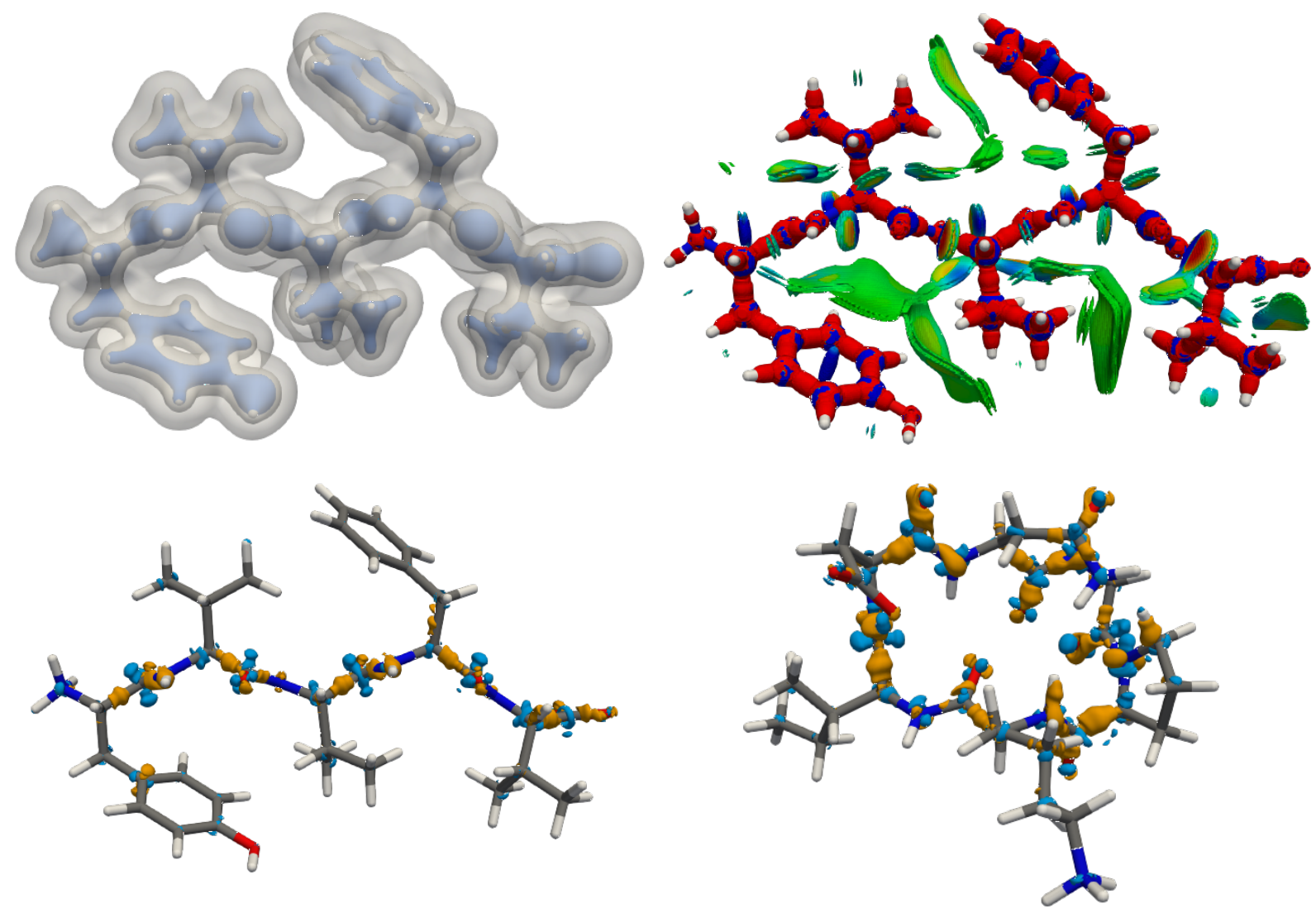

Figure 8: (top left) predicted electron density of enkephalin (PBD ID: 4OLR) at three isovalues: $0.25,0.1$, and $0.01 \mathrm{e}^{-} \mathrm{Bohr}^{-3}$. (top right) DORI map of enkephalin (DORI isovalue: 0.9 ) colored by $\operatorname{sgn}\left(\lambda_{2}\right) \rho(\boldsymbol{r})$ in the range from -0.02 a.u. (red) to 0.02 a.u. (blue) (lower left) density difference between predicted and ab-initio electron density (isovalues $\pm 0.01 \mathrm{e}^{-}$Bohr $\left.^{-3}\right)$. (lower right) density difference between predicted and ab-initio electron density of $3 \mathrm{WNE}$ (isovalues $\pm 0.01 \mathrm{e}^{-} \mathrm{Bohr}^{-3}$ ).

Although similar chemical environments were included in the training set, the error is determined by the lack of an explicit peptide bond motif and cyclopeptides in the training set. While this limitation could be addressed by ad hoc modification of the training set, the overall performance of the machine-learning model is rather exceptional as it provides in only a few minutes electron densities of DFT quality for large and complex molecular 
systems.

\section{Conclusion}

Given its central role in electronic structure methods, the total electron density is a very promising target for machine learning, since accurate predictions of $\rho(\mathbf{r})$ give access to all the information needed to characterize a chemical system. Among the many possible properties that can be computed from the electron density, the patterns arising from non-covalent interactions constitute a particular challenge for machine learning models owing to their longrange nature and subtle physical origin. An effective ML model should be transferable across different systems, efficient in learning from relatively small training sets, and accurate in predicting a $\rho(\mathbf{r})$ both in the quickly-varying region around the atomic nuclei, in the tail and - crucially for the study of non-covalent interactions - that are highly non-local and manifest in real space as slowing-varying regions with low densities. In this work we have presented a model that fulfills all of these requirements, based on an atom-centered decomposition of the density that uses an accurate resolution-of-identity basis set, a symmetry-adapted Gaussian Process regression ML scheme, and training on a diverse database of 2000 sidechain sidechain dimers extracted from the biofragment database.

The model reaches a $0.3 \%$ accuracy on a validation set, that is sufficient to investigate density-based fingerprints of NCIs, and to evaluate the electrostatic potential with sufficient accuracy to quantitatively estimate residue-residue interactions. The transferability of the model is demonstrated by predicting, at a cost that is orders of magnitude smaller than by explicit electronic structure calculations, the electron density for a demonstrative set of oligopeptides, with an accuracy sufficient to reliably visualize bonding patterns and noncovalent domains using the DORI scalar field. Even though the model reaches an impressive

accuracy (0.5\% mean absolute percentage error) for dimers that are predominantly bound by electrostatic interactions, the comparatively larger error suggests that future work should 
focus on resolving the dichotomy between the local machine learning framework and the long-range nature of the intermolecular interactions.

\section{Acknowledgement}

The National Centre of Competence in Research (NCCR) "Materials' Revolution: Computational Design and Discovery of Novel Materials (MARVEL)" of the Swiss National Science Foundation (SNSF) and the EPFL are acknowledged for financial support. AG acknowledges funding from the Max Planck-EPFL center.

\section{Supporting Information Available}

The Supporting Information

\section{References}

(1) Stone, A. International Series of Monographs on Chemistry; Oxford University Press, 2013.

(2) Buckingham, A. D.; Fowler, P. W.; Hutson, J. M. Theoretical studies of van der Waals molecules and intermolecular forces. Chem. Rev. 1988, 88, 963-988.

(3) Castleman, Jr., A.; Hobza, P. van der Waais Molecules II: Introduction. Chem. Rev. 1994, 94, 1721-1722.

(4) Brutschy, B.; Hobza, P. van der Waals Molecules III: Introduction. Chem. Rev. 2000, 100, 3861-3862.

(5) Hobza, P.; Rezáč, J. Introduction: Noncovalent Interactions. Chem. Rev. 2016, 116, 4911-4912. 
(6) Pastorczak, E.; Corminboeuf, C. Perspective: Found in translation: Quantum chemical tools for grasping non-covalent interactions. J. Chem. Phys. 2017, 146, 120901.

(7) Parr, R.; Weitao, Y. Density-Functional Theory of Atoms and Molecules; International Series of Monographs on Chemistry; Oxford University Press, 1994.

(8) Kohn, W.; Sham, L. J. Self-Consistent Equations Including Exchange and Correlation Effects. Phys. Rev. 1965, 140, A1133-A1138.

(9) Cohen, A. J.; Mori-Sánchez, P.; Yang, W. Challenges for Density Functional Theory. Chem. Rev. 2012, 112, 289-320.

(10) Becke, A. D. Perspective: Fifty years of density-functional theory in chemical physics. J. Chem. Phys. 2014, 140, 18A301.

(11) Mardirossian, N.; Head-Gordon, M. Thirty years of density functional theory in computational chemistry: an overview and extensive assessment of 200 density functionals. Mol. Phys. 2017, 115, 2315-2372.

(12) Porezag, D.; Pederson, M. R. Infrared intensities and Raman-scattering activities within density-functional theory. Physical Review B 1996, 54, 7830-7836.

(13) Gilson, M. K.; Honig, B. H. Calculation of electrostatic potentials in an enzyme active site. Nature 1987, 330, 84-86.

(14) Mecozzi, S.; West, A. P.; Dougherty, D. A. Cation-pi interactions in aromatics of biological and medicinal interest: electrostatic potential surfaces as a useful qualitative guide. Proc. Nati. Acad. Sci. USA 1996, 93, 10566-10571.

(15) Sagara, T.; Klassen, J.; Ganz, E. Computational study of hydrogen binding by metalorganic framework-5. J. Chem. Phys. 2004, 121, 12543.

(16) Cardamone, S.; Hughes, T. J.; Popelier, P. L. A. Multipolar electrostatics. Phys. Chem. Chem. Phys. 2014, 16, 10367. 
(17) Polavarapu, P. L. Ab initio vibrational Raman and Raman optical activity spectra. J. Phys. Chem. 1990, 94, 8106-8112.

(18) Hughes, J. L. P.; Sipe, J. E. Calculation of second-order optical response in semiconductors. Phys. Rev. B 1996, 53, 10751-10763.

(19) Sipe, J. E.; Shkrebtii, A. I. Second-order optical response in semiconductors. Phys. Rev. B 2000, 61, 5337-5352.

(20) Sharma, S.; Ambrosch-Draxl, C. Second-Harmonic Optical Response from First Principles. Phys. Scr. 2004, T109, 128.

(21) Masunov, A. E.; Tannu, A.; Dyakov, A. A.; Matveeva, A. D.; Freidzon, A. Y.; Odinokov, A. V.; Bagaturyants, A. A. First principles crystal engineering of nonlinear optical materials. I. Prototypical case of urea. J. Chem. Phys. 2017, 146, 244104.

(22) Koritsanszky, T. S.; Coppens, P. Chemical Applications of X-ray Charge-Density Analysis. Chem. Rev. 2001, 101, 1583-1628.

(23) Lecomte, C.; Guillot, B.; Muzet, N.; Pichon-Pesme, V.; Jelsch, C. Ultra-high-resolution X-ray structure of proteins. Cell. Mol. Life Sci. 2004, 61, 774-782.

(24) Jayatilaka, D.; Dittrich, B. X-ray structure refinement using aspherical atomic density functions obtained from quantum-mechanical calculations. Acta Crystallogr. A 2008, $64,383-393$.

(25) Schnieders, M. J.; Fenn, T. D.; Pande, V. S.; Brunger, A. T. Polarizable atomic multipole X-ray refinement: application to peptide crystals. Acta Crystallogr. D 2009, 65, $952-965$.

(26) Brunger, A.; Adams, P. Comprehensive Biophysics; Elsevier, 2012; pp 105-115.

(27) Gatti, C.; Macchi, P. In Modern Charge-Density Analysis; Gatti, C., Macchi, P., Eds.; Springer Netherlands: Dordrecht, 2012. 
(28) Bader, R. F. W. A quantum theory of molecular structure and its applications. Chem. Rev. 1991, 91, 893-928.

(29) Bader, R. In Oxford University Press; Matta, C. F., Boyd, R. J., Eds.; Wiley-VCH Verlag GmbH \& Co. KGaA: Weinheim, Germany, 2007.

(30) de Silva, P.; Corminboeuf, C. Simultaneous Visualization of Covalent and Noncovalent Interactions Using Regions of Density Overlap. J. Chem. Theory Comput. 2014, 10, $3745-3756$.

(31) Johnson, E. R.; Keinan, S.; Mori-Sanchez, P.; Contreras-Garcia, J.; Cohen, A. J.; Yang, W. Revealing Noncovalent Interactions. J. Am. Chem. Soc 2010, 132, 64986506.

(32) Contreras-García, J.; Johnson, E. R.; Keinan, S.; Chaudret, R.; Piquemal, J.-P.; Beratan, D. N.; Yang, W. NCIPLOT: A Program for Plotting Noncovalent Interaction Regions. J. Chem. Theory Comput. 2011, 7, 625-632.

(33) Walker, P. D.; Mezey, P. G. Molecular electron density Lego approach to molecule building. J. Am. Ceram. Soc. 1993, 115, 12423-12430.

(34) Walker, P. D.; Mezey, P. G. Ab Initio Quality Electron Densities for Proteins: A MEDLA Approach. J. Am. Chem. Soc. 1994, 116, 12022-12032.

(35) Exner, T. E.; Mezey, P. G. Ab Initio-Quality Electrostatic Potentials for Proteins: An Application of the ADMA Approach. J. Phys. Chem. A 2002, 106, 11791-11800.

(36) Exner, T. E.; Mezey, P. G. Ab initio quality properties for macromolecules using the ADMA approach. J. Comput. Chem. 2003, 24, 1980-1986.

(37) Szekeres, Z.; Exner, T.; Mezey, P. G. Fuzzy fragment selection strategies, basis set dependence and HF-DFT comparisons in the applications of the ADMA method of macromolecular quantum chemistry. Int. J. Quantum Chem. 2005, 104, 847-860. 
(38) Stoll, H.; Wagenblast, G.; Preu $\beta$, H. On the use of local basis sets for localized molecular orbitals. Theor. Chim. Acta 1980, 57, 169-178.

(39) Meyer, B.; Guillot, B.; Ruiz-Lopez, M. F.; Genoni, A. Libraries of Extremely Localized Molecular Orbitals. 1. Model Molecules Approximation and Molecular Orbitals Transferability. J. Chem. Theory Comput. 2016, 12, 1052-1067.

(40) Meyer, B.; Guillot, B.; Ruiz-Lopez, M. F.; Jelsch, C.; Genoni, A. Libraries of Extremely Localized Molecular Orbitals. 2. Comparison with the Pseudoatoms Transferability. J. Chem. Theory Comput. 2016, 12, 1068-1081.

(41) Meyer, B.; Genoni, A. Libraries of Extremely Localized Molecular Orbitals. 3. Construction and Preliminary Assessment of the New Databanks. J. Phys. Chem. A 2018,

(42) Hirshfeld, F. L. Difference densities by least-squares refinement: fumaramic acid. Acta Crystallogr. B 1971, 27, 769-781.

(43) Stewart, R. F. Electron population analysis with rigid pseudoatoms. Acta Crystallogr. A 1976, 32, 565-574.

(44) Hansen, N. K.; Coppens, P. Testing aspherical atom refinements on small-molecule data sets. Acta Crystallogr. A 1978, 34, 909-921.

(45) Pichon-Pesme, V.; Lecomte, C.; Lachekar, H. On Building a Data Bank of Transferable Experimental Electron Density Parameters Applicable to Polypeptides. The Journal of Physical Chemistry 1995, 99, 6242-6250.

(46) Jelsch, C.; Pichon-Pesme, V.; Lecomte, C.; Aubry, A. Transferability of multipole charge-density parameters: application to very high resolution oligopeptide and protein structures. Acta Crystallogr., Sect. D 1998, 54, 1306-1318.

(47) Zarychta, B.; Pichon-Pesme, V.; Guillot, B.; Lecomte, C.; Jelsch, C. On the application 
of an experimental multipolar pseudo-atom library for accurate refinement of smallmolecule and protein crystal structures. Acta Crystallogr., Sect. A 2007, 63, 108-125.

(48) Lecomte, C.; Jelsch, C.; Guillot, B.; Fournier, B.; Lagoutte, A. Ultrahigh-resolution crystallography and related electron density and electrostatic properties in proteins. Journal of Synchrotron Radiation 2008, 15, 202-203.

(49) Domagala, S.; Munshi, P.; Ahmed, M.; Guillot, B.; Jelsch, C. Structural analysis and multipole modelling of quercetin monohydrate a quantitative and comparative study. Acta Crystallogr., Sect. B 2011, 67, 63-78.

(50) Domagala, S.; Fournier, B.; Liebschner, D.; Guillot, B.; Jelsch, C. An improved experimental databank of transferable multipolar atom models ELMAM2. Construction details and applications. Acta Crystallogr., Sect. A 2012, 68, 337-351.

(51) Koritsanszky, T.; Volkov, A.; Coppens, P. Aspherical-atom scattering factors from molecular wave functions. 1. Transferability and conformation dependence of atomic electron densities of peptides within the multipole formalism. Acta Crystallogr., Sect. A 2002, 58, 464-472.

(52) Dominiak, P. M.; Volkov, A.; Li, X.; Messerschmidt, M.; Coppens, P. A Theoretical Databank of Transferable Aspherical Atoms and Its Application to Electrostatic Interaction Energy Calculations of Macromolecules. J. Chem. Theory Comput. 2007, 3, $232-247$.

(53) Dittrich, B.; Koritsnszky, T.; Luger, P. A Simple Approach to Nonspherical Electron Densities by Using Invarioms. Angew. Chem., Int. Ed. 2004, 43, 2718-2721.

(54) Hathwar, V. R.; Thakur, T. S.; Row, T. N. G.; Desiraju, G. R. Transferability of Multipole Charge Density Parameters for Supramolecular Synthons: A New Tool for Quantitative Crystal Engineering. Cryst. Growth Des. 2011, 11, 616-623. 
(55) Brockherde, F.; Vogt, L.; Li, L.; Tuckerman, M. E.; Burke, K.; Müller, K.-R. Bypassing the Kohn-Sham equations with machine learning. Nature Communications 2017, 8, 872 .

(56) Bogojeski, M.; Brockherde, F.; Vogt-Maranto, L.; Li, L.; Tuckerman, M. E.; Burke, K.; Müller, K.-R. Efficient prediction of 3D electron densities using machine learning. arXiv:1811.06255 2018,

(57) Grisafi, A.; Wilkins, D. M.; Csányi, G.; Ceriotti, M. Symmetry-Adapted Machine Learning for Tensorial Properties of Atomistic Systems. Phys. Rev. Lett. 2018, 120, 036002.

(58) Grisafi, A.; Fabrizio, A.; Meyer, B.; Wilkins, D. M.; Corminboeuf, C.; Ceriotti, M. Transferable Machine-Learning Model of the Electron Density. ACS Centr. Sci. 2019, $5,57-64$.

(59) Alred, J. M.; Bets, K. V.; Xie, Y.; Yakobson, B. I. Machine learning electron density in sulfur crosslinked carbon nanotubes. Compos. Sci. Technol. 2018, 166, 3-9.

(60) Chandrasekaran, A.; Kamal, D.; Batra, R.; Kim, C.; Chen, L.; Ramprasad, R. Solving the electronic structure problem with machine learning. npj Comput. Mater. 2019, 5, 22.

(61) Fowler, A. T.; Pickard, C. J.; Elliott, J. A. Managing uncertainty in data-derived densities to accelerate density functional theory. J. Phys. Mater. 2019, 2, 034001.

(62) Jurečka, P.; Šponer, J.; Černý, J.; Hobza, P. Benchmark database of accurate (MP2 and $\operatorname{CCSD}(\mathrm{T})$ complete basis set limit) interaction energies of small model complexes, DNA base pairs, and amino acid pairs. Phys. Chem. Chem. Phys. 2006, 8, 1985-1993.

(63) Zhao, Y.; Truhlar, D. G. Density Functionals with Broad Applicability in Chemistry. Acc. Chem. Res. 2008, 41, 157-167. 
(64) Řzeáč, J.; Riley, K. E.; Hobza, P. S66: A well-balanced database of benchmark interaction energies relevant to biomolecular structures. J. Chem. Theory Comput. 2011, 7, $2427-2438$.

(65) Burns, L. A.; Vázquez-Mayagoitia, Á.; Sumpter, B. G.; Sherrill, C. D. Densityfunctional approaches to noncovalent interactions: A comparison of dispersion corrections (DFT-D), exchange-hole dipole moment (XDM) theory, and specialized functionals. J.Chem Phys. 2011, 134, 084107.

(66) Marshall, M. S.; Burns, L. A.; Sherrill, C. D. Basis set convergence of the coupled-cluster correction, MP2CCSD(T): Best practices for benchmarking non-covalent interactions and the attendant revision of the S22, NBC10, HBC6, and HSG databases. J. Chem. Phys. 2011, 135, 194102.

(67) Risthaus, T.; Grimme, S. Benchmarking of London Dispersion-Accounting Density Functional Theory Methods on Very Large Molecular Complexes. Journal of chemical theory and computation 2013, 9, 1580-91.

(68) Schneebeli, S. T.; Bochevarov, A. D.; Friesner, R. A. Parameterization of a B3LYP specific correction for noncovalent interactions and basis set superposition error on a gigantic data set of $\operatorname{CCSD}(\mathrm{T})$ quality noncovalent interaction energies. J. Chem. Theory Comput. 2011, 7, 658-668.

(69) Mardirossian, N.; Head-Gordon, M. $\omega$ B97M-V: A combinatorially optimized, rangeseparated hybrid, meta-GGA density functional with VV10 nonlocal correlation. J. Chem. Phys. 2016,

(70) Burns, L. A.; Faver, J. C.; Zheng, Z.; Marshall, M. S.; Smith, D. G.; Vanommeslaeghe, K.; MacKerell, A. D.; Merz, K. M.; Sherrill, C. D. The BioFragment Database (BFDb): An open-data platform for computational chemistry analysis of noncovalent interactions. J. Chem. Phys. 2017, 147, 161727. 
(71) Grisafi, A.; Wilkins, D. M.; Willatt, M. J.; Ceriotti, M. Atomic-scale representation and statistical learning of tensorial properties. arXiv:1904.01623 2019,

(72) Helgaker, T.; Jørgensen, P.; Olsen, J. Molecular Electronic-Structure Theory; John Wiley \& Sons, Ltd: Chichester, UK, 2000.

(73) Whitten, J. L. Coulombic potential energy integrals and approximations. J. Chem. Phys. 1973, 58, 4496-4501.

(74) Dunlap, B. I.; Connolly, J. W. D.; Sabin, J. R. On the applicability of LCAO-X $\alpha$ methods to molecules containing transition metal atoms: The nickel atom and nickel hydride. Int. J. Quantum Chem. Symp. 1977, 11, 81-87.

(75) Feyereisen, M.; Fitzgerald, G.; Komornicki, A. Use of approximate integrals in ab initio theory. An application in MP2 energy calculations. Chem. Phys. Lett. 1993, 208, 359363.

(76) Rendell, A. P.; Lee, T. J. Coupledcluster theory employing approximate integrals: An approach to avoid the input/output and storage bottlenecks. J. Chem. Phys. 1994, 101, 400-408.

(77) Eichkorn, K.; Treutler, O.; Öhm, H.; Häser, M.; Ahlrichs, R. Auxiliary basis sets to approximate Coulomb potentials. Chem. Phys. Lett. 1995, 240, 283-290.

(78) Weigend, F. A fully direct RI-HF algorithm: Implementation, optimised auxiliary basis sets, demonstration of accuracy and efficiency. Phys. Chem. Chem. Phys. 2002, 4, $4285-4291$.

(79) Werner, H.-J.; Manby, F. R.; Knowles, P. J. Fast linear scaling second-order MøllerPlesset perturbation theory (MP2) using local and density fitting approximations. $J$. Chem. Phys. 2003, 118, 8149-8160. 
(80) Kohn, W. Density Functional and Density Matrix Method Scaling Linearly with the Number of Atoms. Phys. Rev. Lett. 1996, 76, 3168-3171.

(81) Prodan, E.; Kohn, W. Nearsightedness of electronic matter. Proc. Nati. Acad. Sci. USA 2005, 102, 11635-11638.

(82) NAGY, A.; MARCH, N. H. Ratio of density gradient to electron density as a local wavenumber to characterize the ground state of spherical atoms. Mol. Phys. 1997, 90, $271-276$.

(83) Bohórquez, H. J.; Boyd, R. J. On the local representation of the electronic momentum operator in atomic systems. J. Chem. Phys. 2008, 129, 024110.

(84) Nagy, Á.; Liu, S. Local wave-vector, Shannon and Fisher information. Phys. Lett. A 2008, 372, 1654-1656.

(85) Murray, J. S.; Brinck, T.; Lane, P.; Paulsen, K.; Politzer, P. Statistically-based interaction indices derived from molecular surface electrostatic potentials: a general interaction properties function (GIPF). J. Mol. Struct. 1994, 30\%, 55-64.

(86) Murray, J. S.; Politzer, P. Statistical analysis of the molecular surface electrostatic potential: an approach to describing noncovalent interactions in condensed phases. $J$. Mol. Struct. 1998, 425, 107-114.

(87) Bootsma, A. N.; Doney, A. C.; Wheeler, S. Predicting the Strength of Stacking Interactions Between Heterocycles and Aromatic Amino Acid Side Chains. 2019,

(88) Bootsma, A. N.; Wheeler, S. Converting SMILES to Stacking Interaction Energies. 2019 ,

(89) Volkov, A.; Koritsanszky, T.; Coppens, P. Combination of the exact potential and multipole methods (EP/MM) for evaluation of intermolecular electrostatic interaction 
energies with pseudoatom representation of molecular electron densities. Chem. Phys. Lett. 2004, 391, 170-175.

(90) Bootsma, A. N.; Wheeler, S. E. Tuning Stacking Interactions between AspArg Salt Bridges and Heterocyclic Drug Fragments. J. Chem. Inf. Model. 2019, 59, 149-158.

(91) Berman, H. M. The Protein Data Bank. Nucleic Acids Res. 2000, 28, 235-242. 
